\title{
Erythropoietin inhibits SGK1-dependent Th17 cell induction and Th17 cell- dependent kidney disease
}

Chiara Donadei, ${ }^{1,2}$ Andrea Angeletti, ${ }^{1,2}$ Chiara Cantarelli, ${ }^{1,3}$ Vivette D. D'Agati, ${ }^{4}$ Gaetano La Manna, ${ }^{2}$ Enrico Fiaccadori, ${ }^{3}$ Julian K. Horwitz, ${ }^{1}$ Huabao Xiong, ${ }^{1}$ Chiara Guglielmo, ${ }^{1}$ Susan Hartzell, ${ }^{1}$ Joren C. Madsen, ${ }^{5}$ Umberto Maggiore, ${ }^{3}$ Peter S. Heeger, ${ }^{1}$ and Paolo Cravedi'

'Department of Medicine, Translational Transplant Research Center, Precision Institute of Immunology, Icahn School of Medicine at Mount Sinai, New York, New York, USA. ${ }^{2}$ Nephrology Dialysis and Renal Transplantation Unit, S. Orsola University Hospital, Bologna, Italy. ${ }^{3}$ Dipartimento di Medicina e Chirurgia (Università di Parma), UO Nefrologia (Azienda Ospedaliera-Universitaria Parma), Parma, Italy. ${ }^{4}$ Department of Pathology, College of Physicians and Surgeons of Columbia University, New York, New York, USA. ${ }^{5}$ Center for Transplantation Sciences and Division of Cardiac Surgery, Department of Surgery, Massachusetts General Hospital, Boston, Massachusetts, USA.

IL-17-producing CD4+ (Th17) cells are pathogenically linked to autoimmunity and, specifically, to autoimmune kidney disease. The newly recognized immunoregulatory functions of erythropoietin (EPO) and its predominant intrarenal source suggested that EPO physiologically regulates Th17 cell differentiation, thereby serving as a barrier to development of autoimmune kidney disease. Using in vitro studies of human and murine cells and in vivo models, we show that EPO ligation of its receptor (EPO-R) on CD4 $4^{+} T$ cells directly inhibits Th17 cell generation and promotes transdifferentiation of Th17 cells into IL-17-FOXP3 ${ }^{+} \mathrm{CD}^{+} \mathrm{T}$ cells. Mechanistically, EPO/EPO-R ligation abrogates upregulation of SGK1 gene expression and blocks $p 38$ activity to prevent SGK1 phosphorylation, thereby inhibiting RORC-mediated transcription of IL17 and IL23 receptor genes. In a murine model of Th17 cell-dependent aristolochic acid-induced interstitial kidney disease associated with reduced renal EPO production, we demonstrate that transgenic EPO overexpression or recombinant EPO (rEPO) administration limits Th17 cell formation and clinical/histological disease expression. EPO/EPO-R ligations on $\mathrm{CD}^{+} \mathrm{T}$ cells abrogate, while absence of $\mathrm{T}$ cellexpressed EPO-R augments, Th17 cell induction and clinical/histological expression of pristaneinduced glomerulonephritis (associated with decreased intrarenal EPO). rEPO prevents spontaneous glomerulonephritis and Th17 cell generation in MRL-Ipr mice. Together, our findings indicate that EPO physiologically and therapeutically modulates Th17 cells to limit expression of Th17 cellassociated autoimmune kidney disease.

Authorship note: $\mathrm{CD}, \mathrm{AA}$, and $\mathrm{CC}$ contributed equally to this work.

Conflict of interest: The authors have declared that no conflict of interest exists.

Copyright: (c) 2019 American Society for Clinical Investigation

Submitted: January 26, 2019

Accepted: April 12, 2019

Published: April 23, 2019.

Reference information: /CI Insight. 2019;4(10):e127428. https://doi. org/10.1172/ji.insight.127428.

\section{Introduction}

According to current concepts, in mucosal tissues continuously exposed to commensal microbes, e.g., the gastrointestinal tract, the genitourinary tract, and the pulmonary system, immune quiescence is constitutively maintained through innate and adaptive immune mechanisms that include the preferential generation of Tregs $(1,2)$. In the gastrointestinal tract, this Treg-dependent immunoregulation requires local production of retinoic acid and/or TGF- $\beta$ (3) to drive FOXP3 expression in differentiating Tregs. In these organs, infection with pathogenic microbes shifts the immune response toward IL-17 and IL-23 production, a process that is dependent on IL-6/TGF- $\beta$-induced upregulation of transcription factor retinoic acid-related orphan receptor $\gamma \mathrm{T}$ (ROR $\gamma \mathrm{t}$ ) within the T cells. The induced IL-17-producing CD4+ (Th17) cell responses facilitate neutrophil recruitment, among other mechanisms, to promote pathogen clearance (3). In these mucosal sites, once the infection has resolved, the proinflammatory immune response contracts and the immune regulation is restored. While many mechanisms underlying gastrointestinal immunoregulation are understood (1), whether and how they apply to immune responses within the kidney remain unclear.

It is also increasingly recognized that aberrant Th17 cell responses can develop within mucosal organs and, as a consequence, can cause organ inflammation, injury, and dysfunction. Examples include 
experimental colitis (4), autoimmune lung disease (5), lung transplant rejection (6), and various forms of immune-mediated kidney disease, e.g., experimental glomerulonephritis (7), murine models of lupus nephritis (8), and toxin-induced interstitial nephritis (9). Whether and, if so, how local immunoregulatory mechanisms are compromised in these disease processes remains unclear.

Erythropoietin (EPO) is a protein predominantly produced by the kidney in adults and is required for red blood cell production $(10,11)$. EPO is also produced by monocytes $(12)$ and has increasingly been recognized to exhibit nonerythropoietic effects, including immune modulation $(12,13)$. In a previous work, we added to the mechanistic understanding that links EPO to immunoregulation by demonstrating that EPO ligates T cell-expressed EPO receptors (EPO-R), uncouples IL-2 receptor signaling, and thereby inhibits conventional $\mathrm{T}$ cell proliferation and Th1 differentiation (14). In separate studies, we showed that EPO promotes Treg induction and expansion via stimulating release and activation of TGF- $\beta$ by antigen-presenting cells (APCs), which, in turn, induces conversion of naive $\mathrm{CD}^{+} \mathrm{T}$ cells into induced Tregs and promotes kidney transplant acceptance $(14,15)$.

Intriguingly, EPO is often reduced in Th17 cell-associated immune-mediated kidney diseases $(16,17)$. We hypothesized that the immunoregulatory functions of EPO locally and crucially modulate Th17 cell differentiation and the development and/or severity of IL-17-associated disease processes. Herein, we used in vitro systems with human and murine cells as well as multiple in vivo Th17 cell-dependent murine models to test this hypothesis.

\section{Results}

EPO directly inhibits Th17 cell differentiation in vitro. Building upon our previous documentation that EPO inhibits T cell proliferation (14), we used in vitro and in vivo human and murine systems to test whether EPO functions via a distinct mechanism to directly inhibit differentiation of Th17 cells. We stimulated human naive $\mathrm{CD}^{2} 5 \mathrm{RA}^{+} \mathrm{CD} 45 \mathrm{RO}^{-} \mathrm{CD}^{+} \mathrm{T}$ cells with anti-CD3/anti-CD28 mAb in Th17 cell-polarizing conditions with or without recombinant EPO (rEPO) and measured RORC and ILI7 gene expression 24 hours later. While the mRNAs encoding for these gene products were not detectable in naive $\mathrm{CD}^{+} \mathrm{T}$ cells (data not shown) and were markedly upregulated upon exposure to Th17 cell-polarizing conditions, we observed that addition of rEPO significantly inhibited these induced changes (Figure 1, A and B). To test the effects of EPO under stronger Th17 cell-inducing conditions, we exposed the T cells to increasing concentrations of $\mathrm{NaCl}$ (or urea as an osmotic control), a stimulus that was previously shown to augment Th17 cell polarization $(18,19)$. Whereas addition of $\mathrm{NaCl}$ (but not urea) to the cultures augmented RORC and IL17 gene expression, rEPO blunted the increases (Figure 1, A and B). EPO analogously and significantly reduced frequencies of IL-17-producing Th17 cells analyzed on day 5, in the presence or absence of elevated $\mathrm{NaCl}$ concentrations (Figure 1, C and D). To exclude the possibility that reduced Th17 cell induction with $\mathrm{rEPO}$ was mediated by $\mathrm{T}$ cell apoptosis and death, we stained cells for annexin V and 7-AAD (Figure $1 \mathrm{E}$ and Supplemental Figure 1; supplemental material available online with this article; https://doi. org/10.1172/jci.insight.127428DS1). These analyses showed no differences in cell viability and apoptosis, supporting the conclusion that rEPO inhibits Th17 cell differentiation without affecting cell survival.

EPO-induced inhibition of Th17 cell induction associates with decreased p38 and SGK1 phosphorylation. We hypothesized that EPO ligation of EPO-R on the responding T cells inhibits signals that induce upregulation of ROR $\gamma$ t and IL-17 under Th17 cell-polarizing conditions. We crossed EPO-R ${ }^{\mathrm{f} / \mathrm{fl}}$ mice to a mouse in which the Cre recombinase transgene is expressed under the CD4 promoter (CD4-Cre), yielding animals in which a functional EPO-R is absent from all $\mathrm{T}$ cells (verification shown in Supplemental Figure 2). Whereas rEPO inhibited IL-17 production of purified, murine, naive EPO-R ${ }^{\mathrm{f} / \mathrm{fl}} \mathrm{CD} 44^{\mathrm{lo}} \mathrm{CD} 62 \mathrm{~L}^{\mathrm{hi}} \mathrm{CD} 4^{+} \mathrm{T}$ cells (confirming that the findings in human cells from Figure 1, A-D, apply to murine T cells), rEPO had no statistically significant effect on IL-17 production in EPO- $\mathrm{R}^{\mathrm{fl} / \mathrm{fl}} \times \mathrm{CD} 4-\mathrm{Cre}^{+}$naive $\mathrm{CD} 4^{+} \mathrm{T}$ cells cultured under Th17 cell-differentiating conditions (Figure 1, F and G). These results indicate that EPO directly inhibits Th17 cell differentiation through signals transmitted by the T cell-expressed EPO-R.

Current concepts indicate that phosphorylation of SGK1 (20), a serine-threonine kinase that phosphorylates the transcription factor forkhead box protein O1 (FOXO1), is crucial for Th17 cell induction. Phospho-SGK1 (pSGK1) abrogates the suppressive effects of FOXO1 on ROR $\gamma \mathrm{t}$-mediated Il23r expression, thereby enhancing IL-23R expression and Th17 cell stability (20). Sodium increases Th17 cell induction by promoting transcription of $S g k 1$ gene and SGK1 protein phosphorylation (19). To test whether EPO affects Th17 cell induction by counteracting $S G K 1$ transcription and phosphorylation, we cultured human 
A

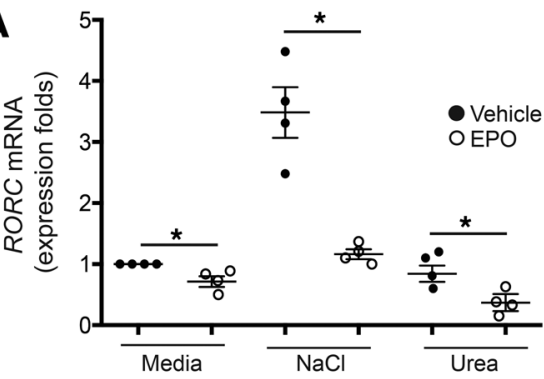

C

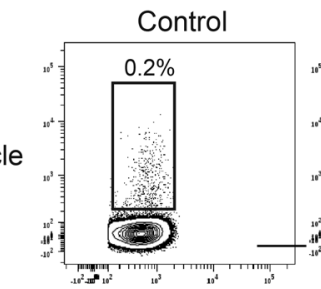

$\mathrm{NaCl}(40 \mathrm{mM})$
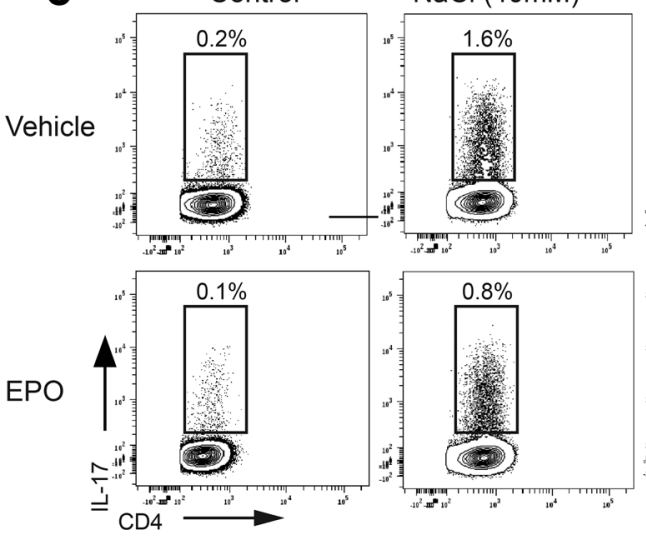

E

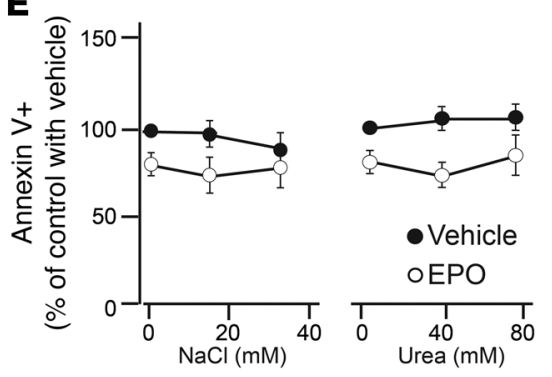

G

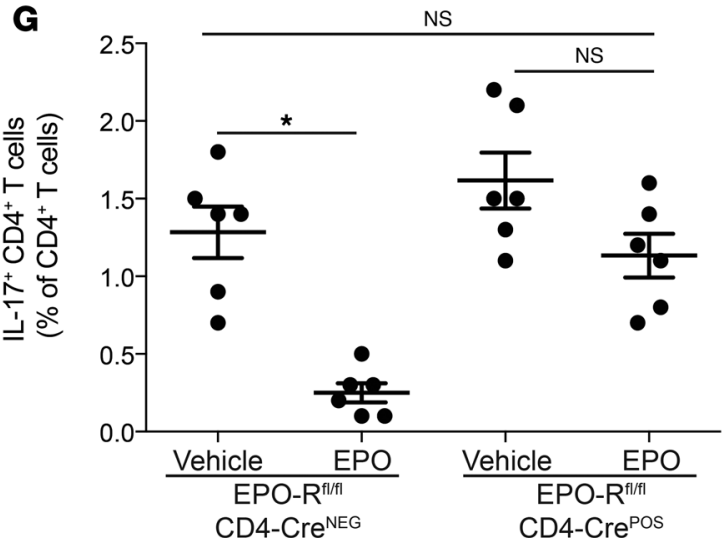

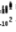

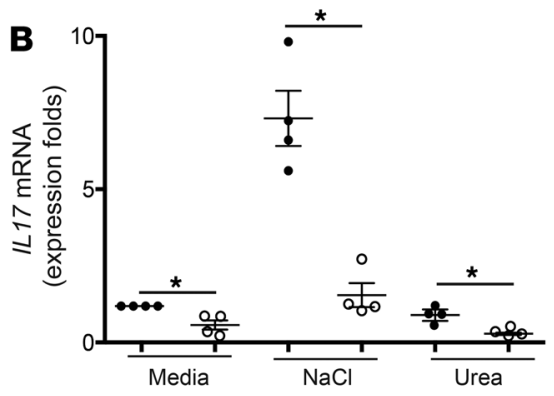

D
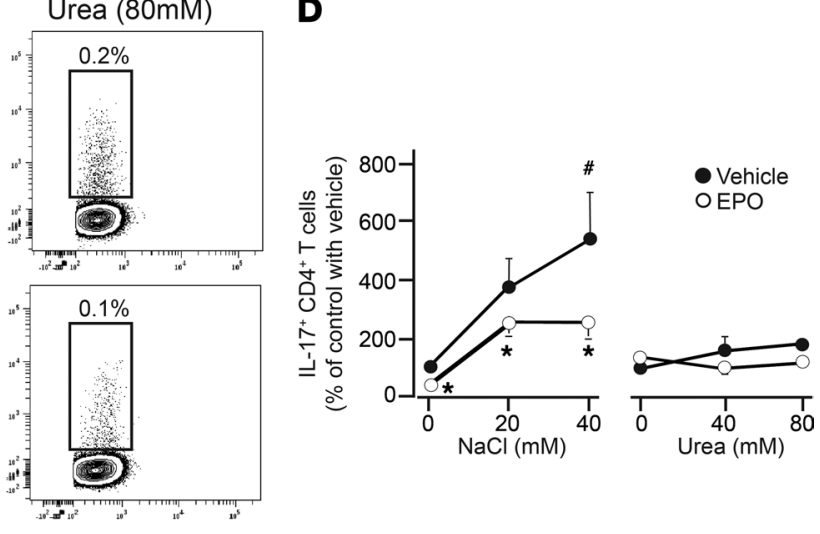

$\mathbf{F}$

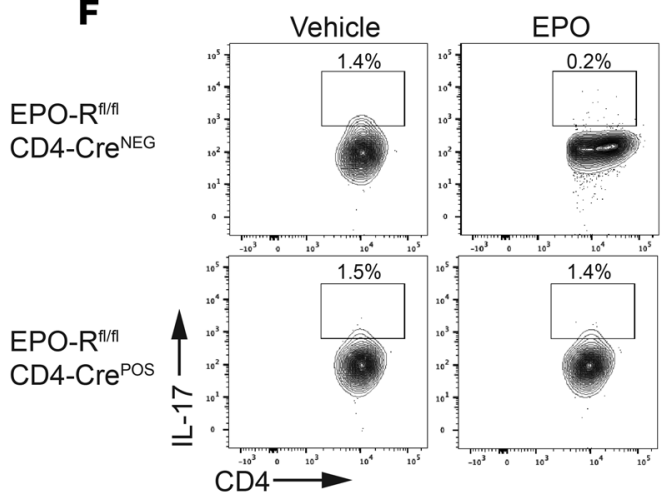

Figure 1. EPO inhibits Th17 cell induction in vitro. Enriched human naive $\mathrm{CD} 4^{+} \mathrm{T}$ cells were cultured in the presence of Th17 cell-polarizing conditions (see Methods) in control media or in media with $20-40 \mathrm{mM}$ $\mathrm{NaCl}$ or $40-80 \mathrm{mM}$ urea added in the presence of EPO $(1000 \mathrm{lU} / \mathrm{ml})$ or vehicle control. (A) RORC and (B) IL17 gene expression ( $n=3$ donors) after 24 hours of culture. ${ }^{*} P<0.05$, paired $t$ test. (C) Representative plots and (D) normalized data quantification of IL-17+CD4+ Th17 cells after 5 days of culture ( 5 experiments from 7 different donors). (E) Quantification of annexin V staining (normalized to vehicle controls) of the cultures in $\mathbf{C}$ and $\mathbf{D}$. Naive $C D 44^{\circ} \mathrm{CD} 62 \mathrm{~L}^{\text {hic }} \mathrm{CD} 4^{+} T$ cells were enriched through negative magnetic isolation from EPO- $R^{\mathrm{fl} / \mathrm{fl}} \mathrm{CD} 4-$ $\mathrm{Cre}^{+}$mice and $\mathrm{Cre}^{-}$controls and were cultured in Th17 cell-polarizing conditions. (F) Representative plots and (C) data quantification of $\mathrm{IL}-17^{+}$cells after 5 days of culture ( $n=6$ mice per group). ${ }^{*} P<0.05$ vs. vehicle; ${ }^{\#}<0.05$ vs. media (no $\mathrm{NaCl}$ or urea), paired $t$ test or 2 -way ANOVA with Tukey test. Data represent mean \pm SEM. 
A

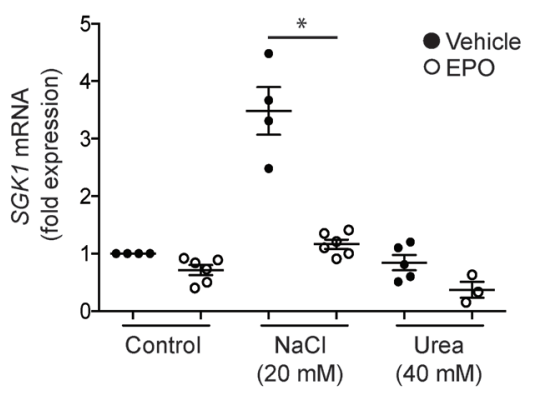

B

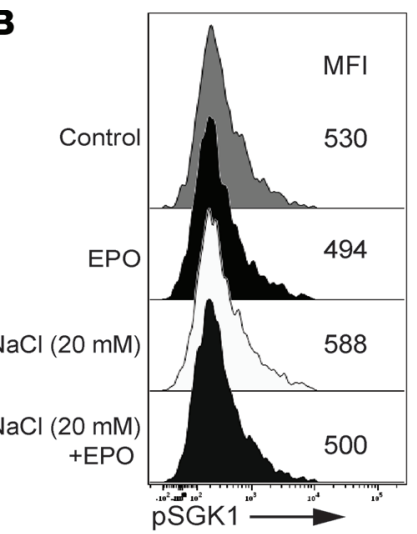

C

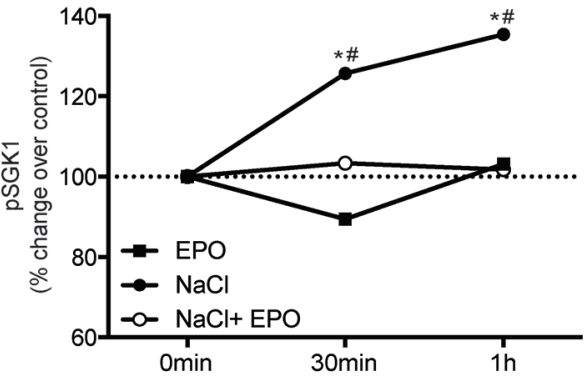

D

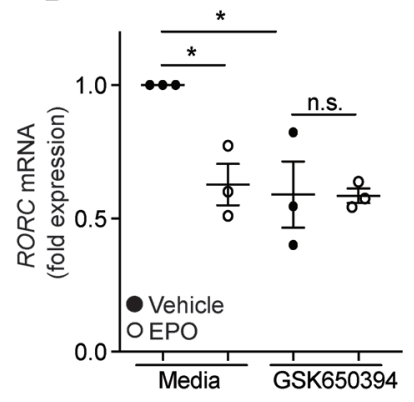

G

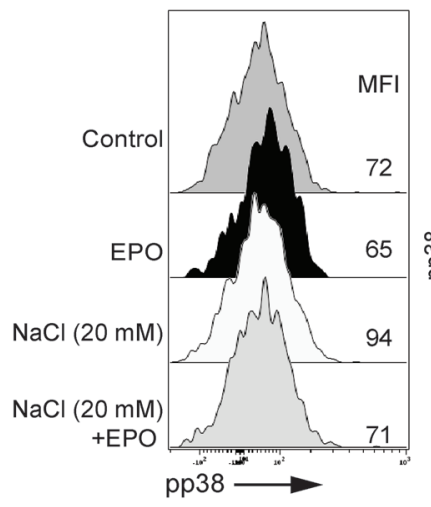

H
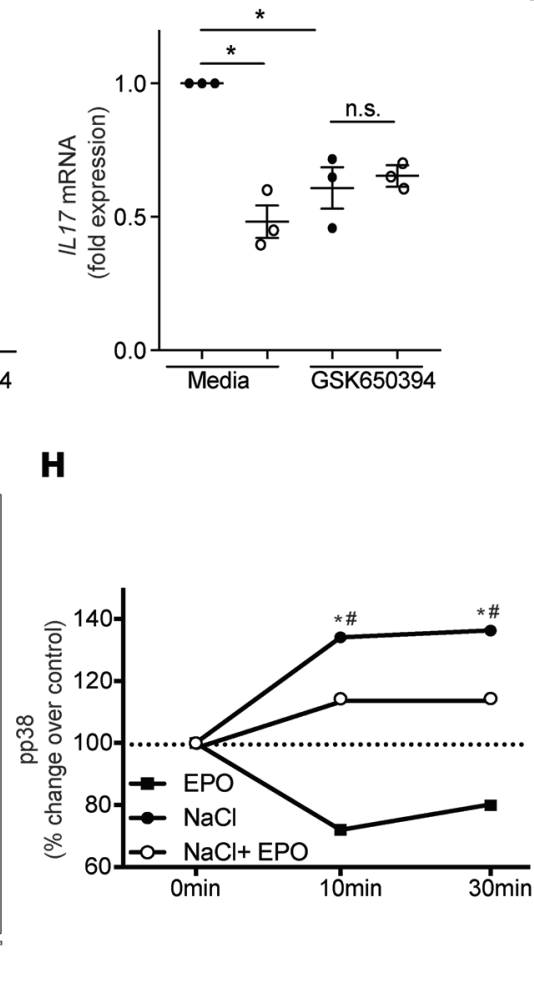

E

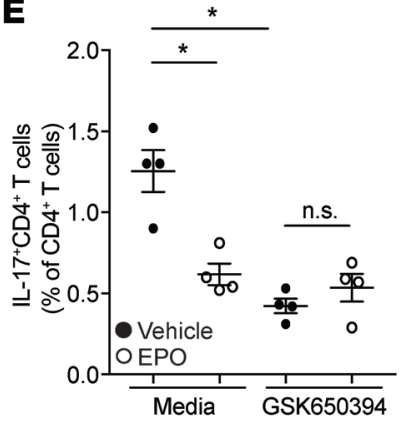

$\mathbf{F}$
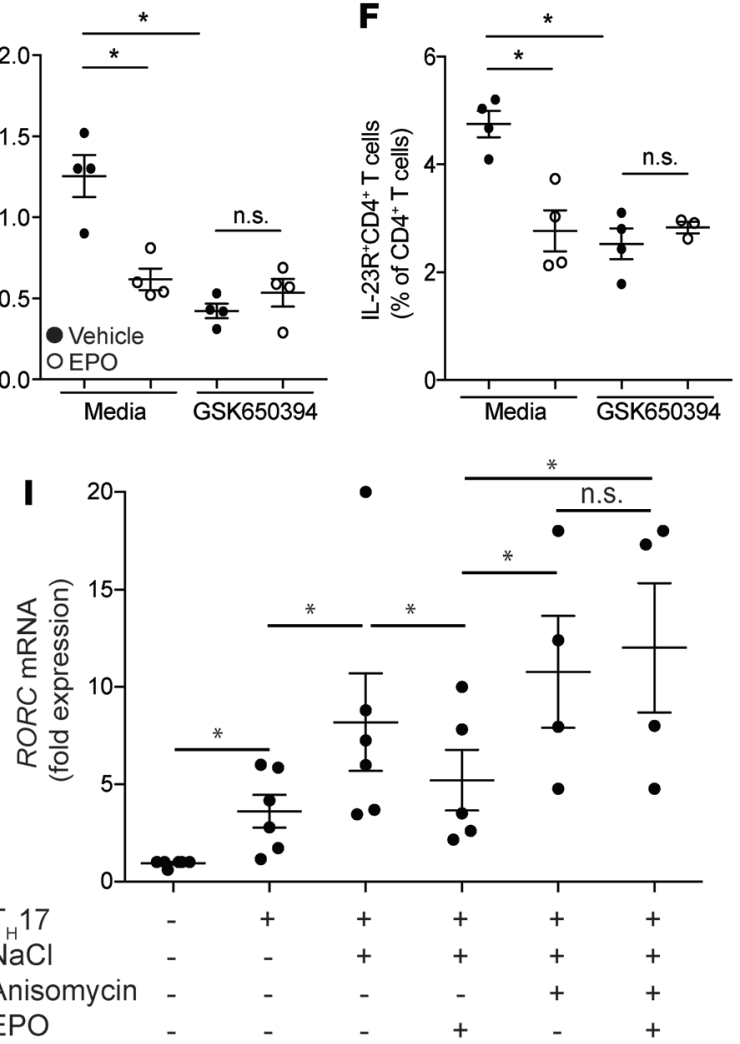

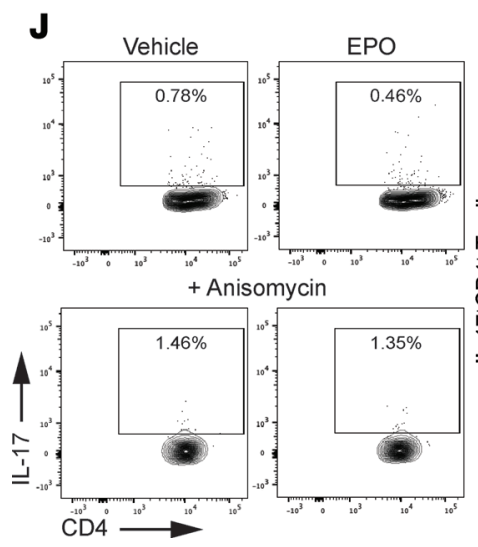

$\mathbf{K}$

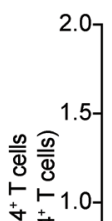

袋势 考苍0.5-

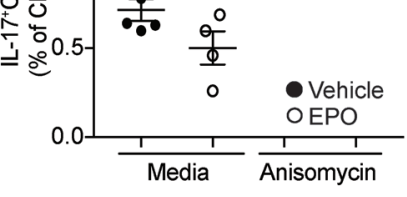

$\mathbf{L}$
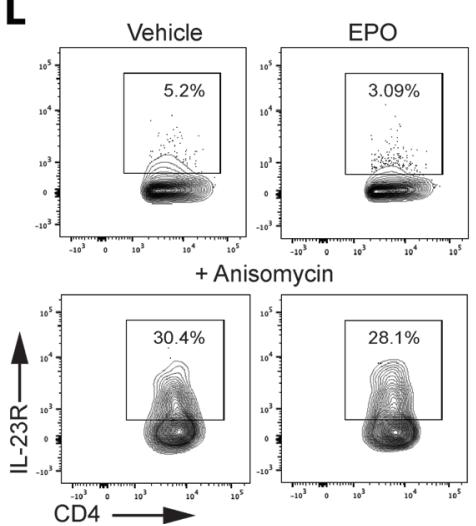

M

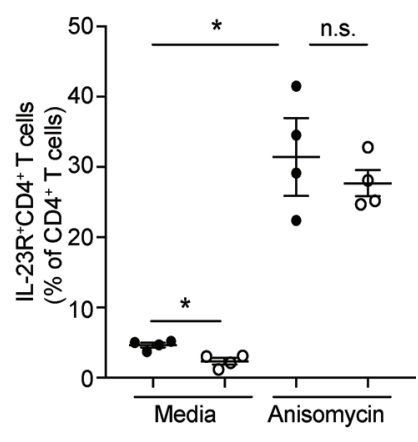


Figure 2. EPO counteracts NaCl-induced p38 activation. (A) SCK1 gene expression in enriched human CD4 ${ }^{+}$T cells activated with anti-CD3/anti-CD28coated beads plus EPO $(1000 \mathrm{IU} / \mathrm{ml})$ or vehicle control in media or with an additional $20-40 \mathrm{mM} \mathrm{NaCl}$ or $40-80$ mM urea added for 24 hours (qRT-PCR). (B) Representative plot and (C) data quantification of pSCK1 expression in enriched human CD4+ $T$ cells activated with anti-CD3/anti-CD28-coated beads plus EPO (1000 IU/ml) or vehicle control for $10-30$ minutes in $40 \mathrm{mM}$ of urea media or $20 \mathrm{mM}$ of NaCl with or without EPO assayed by flow cytometry ( $n$ $=5$ donors). The results are expressed as medians of the percentage of pSCK1 versus unstimulated cells. (D-F) Enriched human naive CD4+ $T$ cells were cultured in Th17 cell-polarizing conditions with or without EPO (1000 IU/mI) and SGK1 inhibitor GSK650394 (10 nM) ( $n=3-4$ donors). (D) RORC and IL17 mRNA at 24 hours. (E) IL-17 and (F) IL-23R expression after 5 days of culture (representative plots in Supplemental Figure 5). (C) Representative plot and (H) data quantification of pp38 expression in enriched human $C D 4^{+} \mathrm{T}$ cells activated, as in $\mathbf{B}$ and $\mathbf{C}$. (I) RORC gene expression in enriched human naive CD4 ${ }^{+}$T cells cultured for 24 hours under Th17 cell-polarizing conditions in standard media or with an additional 20 mM NaCl added with or without EPO and p38 activator anisomycin $(10 \mu \mathrm{g} / \mathrm{ml})(n=7$ donors). (J and $\mathbf{L})$ Representative plots and ( $\mathbf{K}$ and $\mathbf{M})$ data quantification of IL-17 and IL-23R expression in enriched human naive CD4 ${ }^{+} T$ cells cultured under Th17 cell-polarizing conditions for 5 days with or without EPO and p38 activator anisomycin (10 $\left.\mu \mathrm{g} / \mathrm{ml}\right)$ ( $n=5$ donors). ${ }^{*} P<0.05$ vs. NaCl + EPO,${ }^{\#} P<0.05$ vs. 0 min (no NaCl or urea), paired $t$ test or 2 -way ANOVA with Tukey test. Data represent mean \pm SEM.

$\mathrm{T}$ cells with $\mathrm{NaCl}$ (or urea) with or without $\mathrm{EPO}$ for 60 minutes and tested for $S G K 1$ mRNA expression by qRT-PCR (Figure 2A). Whereas $\mathrm{NaCl}$ significantly increased $S G K 1$ mRNA compared with media alone or urea (verifying previously published work by others, ref. 19), EPO prevented the NaCl-induced $S G K 1$ upregulation. NaCl-driven changes in SGK1 gene expression were mirrored by increases in ILI7 and IL23R mRNA, while FOXO1 expression levels diminished (Supplemental Figure 3; see Supplemental Figure 4 for a schematic of the signaling pathway), supporting the previously described link between SGK1 and these mediators of Th17 cell induction (20). EPO-induced inhibition of SGK1 gene expression was associated with reduction of $I L 17$ and $I L 23 R$ and an increase of FOXO1 mRNA, indicating that EPO inhibits SGK1 activation and its linked downstream effectors (Supplemental Figure 3). When we analyzed the kinetics of pSGK1 expression by phospho-flow cytometry, we confirmed that $\mathrm{NaCl}$ significantly increased pSGK1 compared with standard culture media and showed that EPO fully abrogated the $\mathrm{NaCl}$-induced increase in pSGK1 (Figure 2, B and C).

We repeated the Th17 cell induction cultures in the presence or absence of a selective SGK1 antagonist, GSK650394, testing the effects on RORC, IL-17, and IL-23R (Figure 2, D-F, and Supplemental Figure 5). While SGK1 inhibition reduced RORC and IL17 mRNA as well as IL-17 and IL-23R protein, rEPO had no additional inhibitory effects.

EPO inhibitory effects are overcome by $p 38$ and SGK1 antagonism. SGK1 phosphorylation is dependent upon $\mathrm{p} 38$ mitogen-activated protein kinase (20), which is also activated by $\mathrm{NaCl}$ (21). To test whether EPO inhibitory effects on SGK1 are dependent upon inhibition of p38 phosphorylation, we repeated the Th17 cell induction cultures using human $\mathrm{T}$ cells exposed to $\mathrm{NaCl}$ or urea with or without $\mathrm{rEPO}$ and measured phospho-p38 (pp38) levels by flow cytometry (Figure 2, G and H). These analyses showed that EPO fully prevented the $\mathrm{NaCl}$-induced increases in pp38.

To assess functional links among EPO, NaCl-induced pp38, and IL-17 production, we differentiated naive human $\mathrm{CD}^{+} \mathrm{T}$ cells under Th17 cell-polarizing conditions for 48 hours in the presence of $\mathrm{NaCl}$ and EPO, with or without the p38 activator anisomycin (Figure 2I). While rEPO inhibited RORC gene upregulation, the addition of anisomycin overcame these inhibitory effects, increasing $R O R C$ gene expression to levels of control wells without rEPO. To further test the hypothesis that EPO inhibits Th17 cell induction by counteracting p38-induced SGK1 activation, we repeated the Th17 cell induction in the presence or absence of anisomycin and quantified intracellular IL-17 (Figure 2, J and K) and surface expressed IL-23R (Figure $2, \mathrm{~L}$ and $\mathrm{M}$ ) at the end of the culture. These experiments showed that anisomycin markedly upregulated expression of both proteins regardless of the presence of $\mathrm{rEPO}$ in the culture, supporting the conclusion that $\mathrm{EPO} / \mathrm{EPO}-\mathrm{R}$-transmitted signals prevent pp38 activation and, as a consequence, prevent IL-17 and IL-23R upregulation. Together, the data support the conclusion that EPO/EPO-R-initiated signals inhibit Th17 cell differentiation by counteracting activation of p38 (see working model in Supplemental Figure 4).

EPO directly inhibits Th17 cell differentiation in vivo. To test whether EPO inhibits Th17 cell differentiation in vivo independent of any effects of $\mathrm{EPO}$ on Treg generation (15), we isolated naive $\mathrm{CD} 44^{1 \mathrm{lo}} \mathrm{CD} 62 \mathrm{~L}^{\text {hi }} \mathrm{CD} 4^{+}$ $\mathrm{T}$ cells from Foxp $3^{\text {DTR }}$ mice, injected them into rag1 $1^{-/-}$recipients, treated the adoptive hosts with Diphtheria toxin (DT) (Figure 3A), and verified that the DT treatment deleted FOXP3 ${ }^{+}$Tregs in the adoptive hosts 1 week later (Supplemental Figure 6). We then treated the animals with $\mathrm{rEPO}(1000 \mathrm{IU} / \mathrm{kg}$ ) or saline control 3 times/week and analyzed frequencies of cytokine-producing $\mathrm{CD} 4^{+} \mathrm{T}$ cells 6 weeks after the adoptive transfer. These analyses showed that spleens of control animals (DT + vehicle) contained approximately $5 \% \mathrm{Th} 17$ cells. In contrast, we observed a frequency of $<2.5 \% \mathrm{Th} 17$ cells in spleens of the DT + rEPO-treated mice 
A Foxp3 $3^{\mathrm{DTR}}$

CD4 ${ }^{+} T$ cells
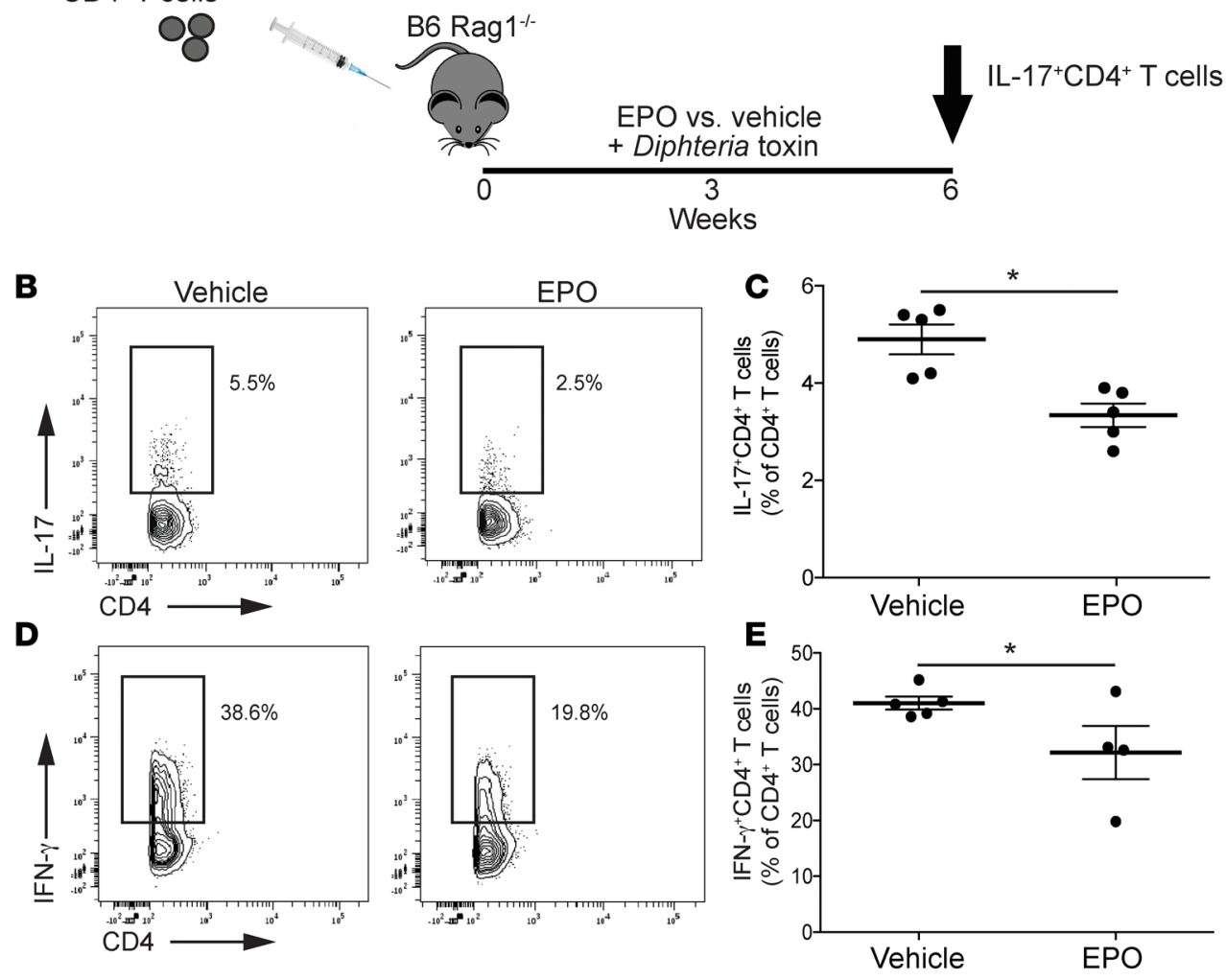

F $\quad$ IL-17 GFP

CD4 ${ }^{+} \mathrm{T}$ cells
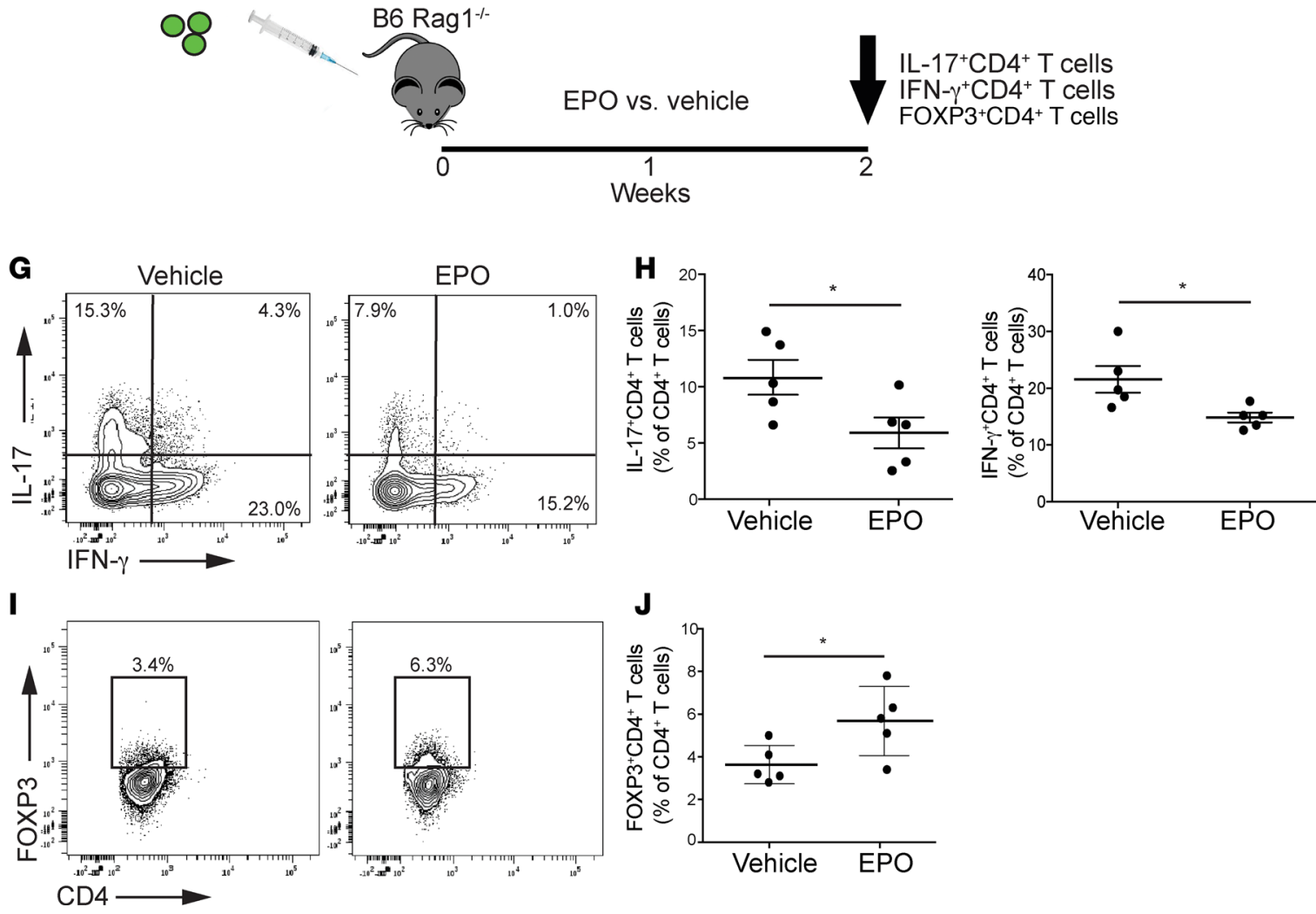
Figure 3. EPO inhibits murine Th17 cell induction and promotes conversion into Tregs in vivo. Rag1/- mice ( $n=5$ per group) were injected with flow-sorted CD44 ${ }^{10} \mathrm{CD} 62 \mathrm{~L}^{\text {hiCD4}}{ }^{+}$T cells from Foxp $3^{\text {DTR }}$ mice previously treated with Diphtheria toxin (10 $\mu \mathrm{g} / \mathrm{kg}$ every other day for 1 week). After CD4 ${ }^{+}$ T cell transfer, rag1//- mice received Diphtheria toxin $(10 \mu \mathrm{g} / \mathrm{kg} 3$ times per week) and either EPO or vehicle control for 6 weeks (A, study design). (B and $\mathbf{D})$ Representative plots and ( $\mathbf{C}$ and $\mathbf{E}$ ) data quantification of CD4+IL-17+ $T$ cells and CD4+IFN- $\gamma^{+}$T cells at 6 weeks. We flow-sorted IL-17-GFP+ cells obtained in vitro by culturing naive CD4 ${ }^{+} \mathrm{T}$ cells from IL-17-GFP mice in Th17 cell-polarizing conditions. These cells were transferred into rag1 ${ }^{-/-}$mice that were treated with EPO of vehicle control (F, study design). ( $\mathbf{G}$ and I) Representative plots and ( $\mathbf{H}$ and $\mathbf{J})$ data quantification of IL-17 ${ }^{+}$and IFN$\gamma^{+} \mathrm{CD} 4^{+} \mathrm{T}$ cells in the spleen at sacrifice. ${ }^{*} P<0.05$, unpaired $t$ test. Data represent mean $\pm \mathrm{SEM}$.

$\left(P<0.05\right.$, Figure 3, B and C). We also observed fewer IFN- $\gamma^{+}$splenic CD $4^{+} \mathrm{T}$ cells in the rEPO-treated mice (Figure 3, D and E). We did not detect $\mathrm{FOXP}^{+}$Tregs in any of the recipients at the time of sacrifice (data not shown). Together, the data support the conclusion that EPO inhibits Th17 cell differentiation in vivo independent of indirect effects mediated via Tregs that we reported previously (15).

EPO promotes conversion of Th17 cells into Ill $-\mathrm{FOXP3}{ }^{+} \mathrm{CD} 4^{+} \mathrm{T}$ cells. As work by others showed that Th17 cells can transdifferentiate into Tregs in vivo $(22,23)$, we next tested the hypothesis that, in addition to directly inhibiting Th17 cell differentiation, EPO converts predifferentiated Th17 cells into FOXP $3^{+} \mathrm{CD} 4^{+}$ $\mathrm{T}$ cells. We isolated naive $\mathrm{CD}^{+} \mathrm{GFP}^{-} \mathrm{T}$ cells from GFP-IL-17 reporter mice and cultured them in vitro under Th17 cell-differentiating conditions. We then flow sorted the induced $\mathrm{GFP}^{+}\left(\mathrm{IL}-17^{+}\right) \mathrm{CD}^{+} \mathrm{T}$ cells (>98\% purity), adoptively transferred them into $\mathrm{rag} 1^{-/-}$recipients, and treated the adoptive hosts with $\mathrm{rEPO}$ or vehicle (Figure $3 \mathrm{~F}$ ) for 7 days $\left(\mathrm{GFP}^{+} \mathrm{IL}-17^{+} \mathrm{CD} 4^{+} \mathrm{T}\right.$ cells were $\mathrm{FOXP3}{ }^{-}$, data not shown). Analyses of the splenic CD4+ ${ }^{+}$cells showed significantly fewer splenic GFP ${ }^{+} \mathrm{IL}-17^{+}$Th17 cells (Figure $3, \mathrm{G}$ and $\mathrm{H}$ ) and significantly more FOXP ${ }^{+} \mathrm{CD}^{+}{ }^{+} \mathrm{T}$ cells in the rEPO-treated mice (Figure $3, \mathrm{I}$ and $\mathrm{J}$ ). As the transferred $\mathrm{CD} 4^{+}$ $\mathrm{T}$ cells in these recipients were initially $\mathrm{IL}-17^{-} \mathrm{GFP}^{+}$, the findings indicate that EPO promotes conversion of Th17 cells into IL-17-FOXP3 ${ }^{+} \mathrm{CD} 4^{+} \mathrm{T}$ cells.

EPO modulates Th17 cell immunity and renal injury induced by aristolochic acid. To test whether kidney-produced EPO influences development/expression of Th17 cell-mediated kidney disease, we employed the aristolochic acid (ArA) model of murine nephropathy. ArA is the etiologic agent of human Balkan or Chinese herb nephropathy, a form of interstitial nephritis manifested as renal insufficiency and urinary protein loss $(24,25)$. Interestingly, ArA nephropathy in humans is strongly associated with low serum EPO levels and with abnormalities in the IL-17/IL-17R axis (26). Administration of ArA to B6 mice mimics many aspects of this human disease (27).

We administered a 3-week course of ArA or vehicle control to B6 mice given a standard diet. Three weeks later ( 6 weeks after initiating the experiment), the animals developed albuminuria and tubular atrophy (Figure 4, A and B), remarkably associated with a downregulation of kidney Epo gene expression and lower serum EPO levels compared with baseline control (Figure 4C). Analysis of kidney mRNA at 3 weeks showed increased Rorc gene expression, with diminished Foxp3 expression compared with controls (Figure 4D). ArA administration augmented frequencies of splenic Th17 cells (Figure 4, E and F) without changing frequencies of FOXP3 ${ }^{+} \mathrm{CD} 4^{+} \mathrm{T}$ cells (Figure 4, G and $\mathrm{H}$ ), reducing the Treg/Th17 cell ratio $(64.2 \pm 31.9$ vs. $12.8 \pm 1.5 ; P<0.05)$. To further increase Th17 cell responses in this system, we fed ArA-treated B6 mice a high- $\mathrm{NaCl}$ diet (supplemented with $4 \% \mathrm{NaCl}$ ) to promote Th17 cell induction. Under these conditions, we observed augmented proteinuria (Figure 4A), tubular atrophy, interstitial fibrosis (Figure 4B), upregulated kidney Rorc gene expression (Figure 4D), and further expanded splenic Th17 cells (without altering frequencies of splenic Tregs) compared with animals on a regular diet (Figure 4, E-H).

To test whether the ArA-induced decreased renal EPO expression is causally linked to Th17 cell development and to the clinical expression of nephritis, we generated mice in which murine EPO was expressed as a doxycycline-driven (DOX-driven) inducible transgene (Epo-Tg rtTA ${ }^{+/-}$mice, Figure 5A). Verifying the model system, oral DOX significantly upregulated kidney Epo gene expression, augmented circulating serum EPO protein, and increased the hematocrit (Figure 5B and Supplemental Figure 7). When we treated Epo-Tg rtTA ${ }^{+}$and control Epo-Tg rtTA ${ }^{-}$mice (which lack the DOX response element) with DOX and ArA, we observed that transgenic overexpression of EPO resulted in less albuminuria (Figure 5C), fewer splenic Th17 cells (Figure 5, D and $\mathrm{E}$ ), and more $\mathrm{FOXP}^{+} \mathrm{CD}^{+} \mathrm{T}$ cells (Figure 5, F and G), leading to a higher Treg/Th17 cell ratio (Figure $5 \mathrm{H}$ ).

Using an alternative therapeutic strategy, we tested the effects of rEPO administration on kidney disease and Th17 cells in ArA-treated mice. We fed groups of ArA-treated WT mice with control versus high- $\mathrm{NaCl}$ diet and administered $\mathrm{rEPO}$ or vehicle (Figure $6 \mathrm{~A}$ ). Increased albuminuria observed in the 
A
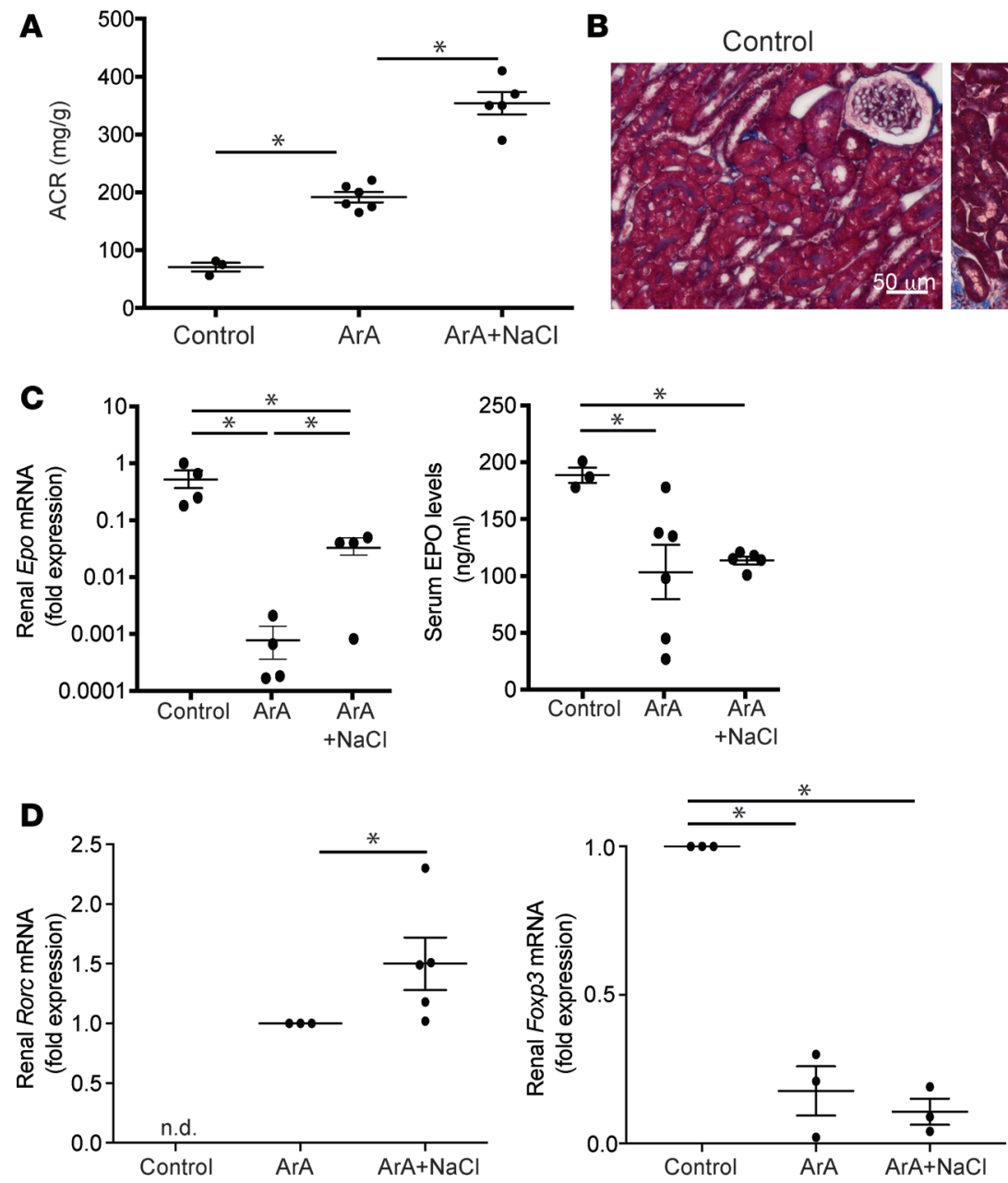

$\operatorname{ArA}$
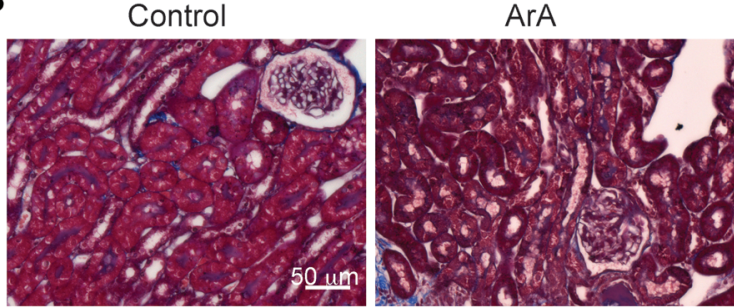

Figure 4. Aristolochic acid induces interstitial nephropathy associated with reduced EPO production and increased Th17 cells in mice. Four- to six-week-old male B6 mice ( $n=3-7$ per group) were given aristolochic acid (ArA) i.p. 3 times per week for 3 weeks and sacrificed after 3 additional weeks ( 6 weeks in total from first ArA administration). During this period, mice were fed a standard or high-NaCl diet. (A) Urinary albumin/creatinine (ACR; by ELISA) and (B) representative Masson's trichrome-stained sections (original magnification, $\times 20$ ) of kidney tissue from control-, ArA-, or ArA plus $\mathrm{NaCl}-$ treated animals. Kidneys from ArA-treated animals show signs of tubular atrophy and interstitial fibrosis (in blue) when given a high-salt diet. (C) Kidney Epo mRNA (qRTPCR, left) and serum EPO protein levels (ELISA, right). (D) Kidney Rorc and Foxp3 mRNA by qRT-PCR. (E) Representative flow cytometry plots and (F) quantified percentages of intracellular IL-17 from PHA/ionomycin-stimulated spleen cells from animals in each group, gated on $\mathrm{CD}^{+}$ T cells. (C) Representative flow cytometry plots and (H) quantified percentages of splenic FOXP3 ${ }^{+} \mathrm{CD} 4^{+} \mathrm{T}$ cells. n.d., not detected. ${ }^{*} P<0.05$, 1-way ANOVA with Tukey test. Data represent mean $\pm \mathrm{SEM}$.
$\mathbf{E}$

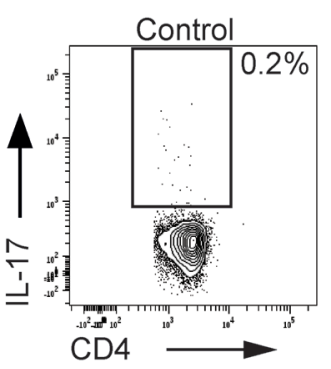

G

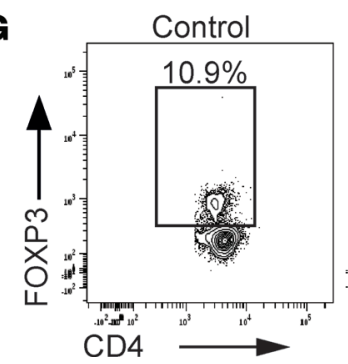

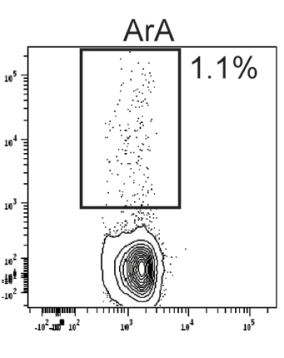
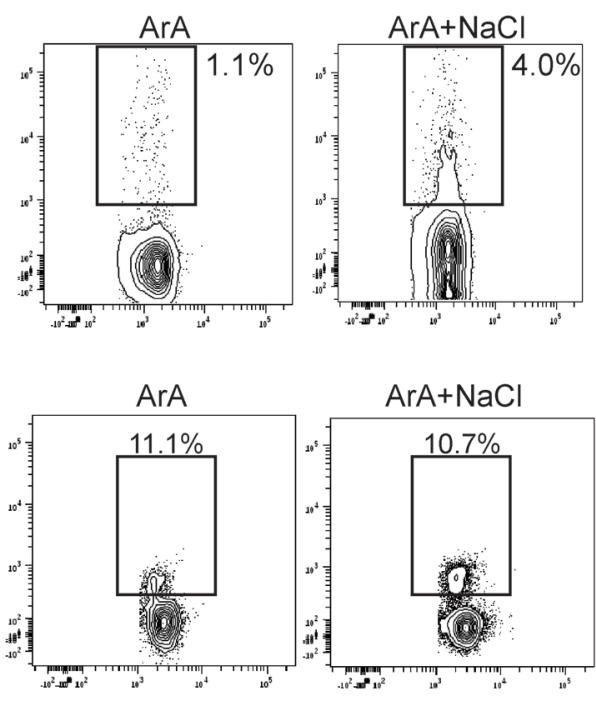

$\mathbf{F}$

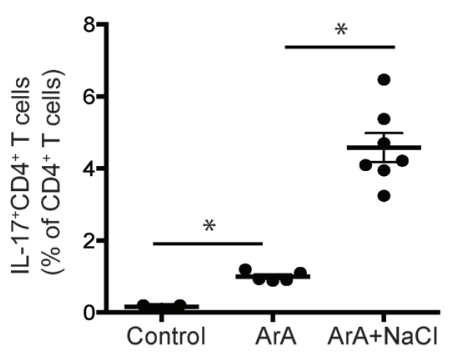

H

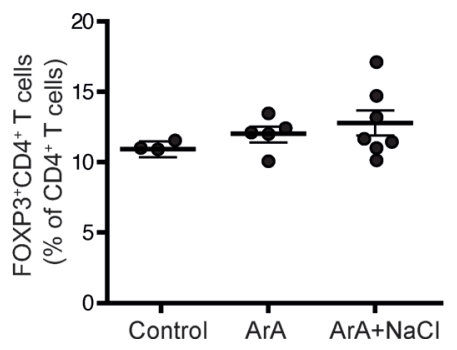

animals fed a high- $\mathrm{NaCl}$ diet was fully prevented by $\mathrm{rEPO}$ (Figure 6B). Administration of $\mathrm{rEPO}$ also counteracted the $\mathrm{NaCl}$-induced changes in renal Rorc and Foxp 3 gene expression (Figure $6 \mathrm{C}$ ) and fully prevented the increase in Th17 cells (Figure 6, D and E), together normalizing the Treg/Th17 cell ratio to control values (Figure 6, F-H). High- $\mathrm{NaCl}$ diet increased IFN- $\gamma$-producing $\mathrm{CD}^{+} \mathrm{T}$ cells in ArA-treated mice (Supplemental Figure 8), which was also prevented by rEPO administration. 
A

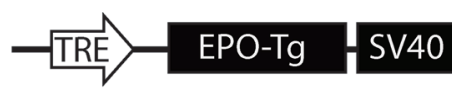

C

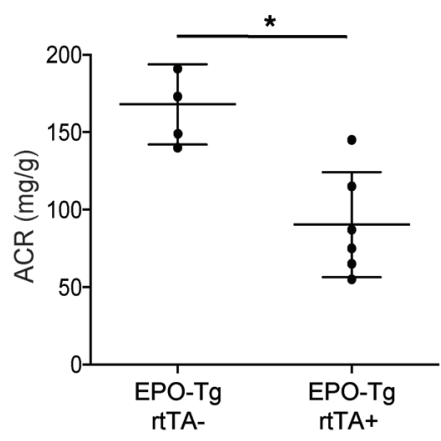

H

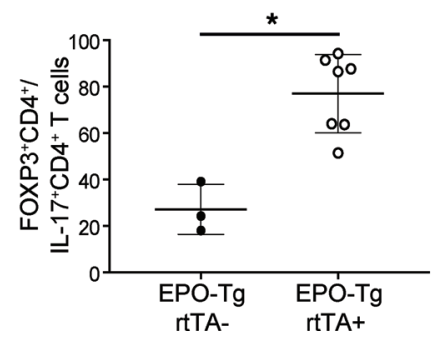

B

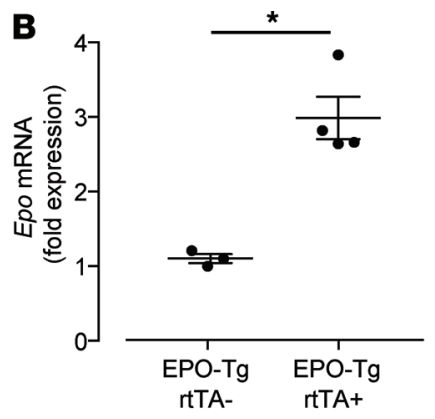

D
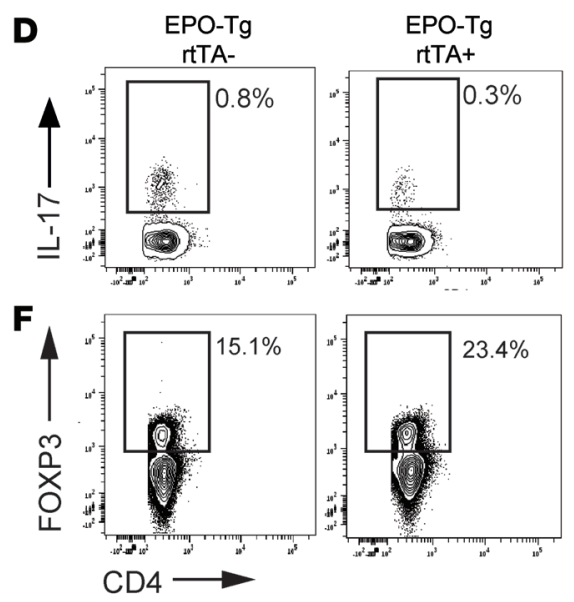
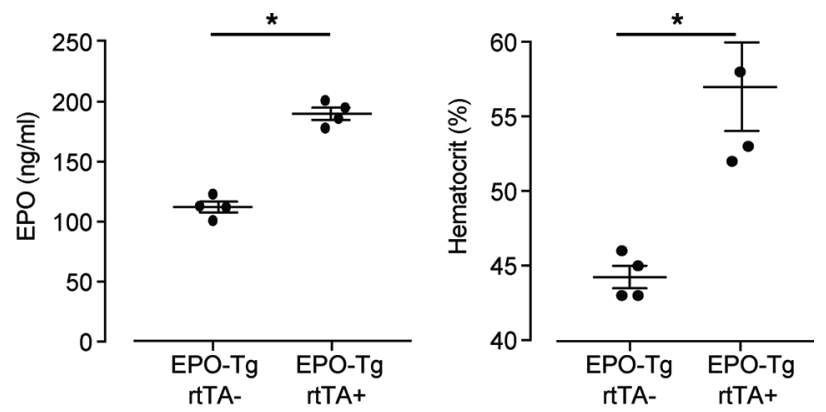

$\mathbf{E}$

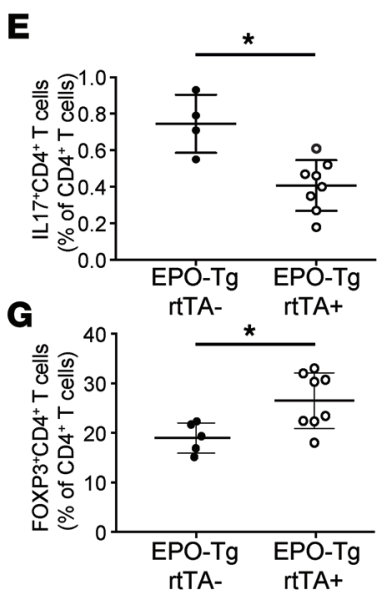

Figure 5. EPO-Tg overexpression ameliorates aristolochic acid nephropathy and reduces Th17 cells. (A) Representative schema of the doxycycline-inducible (DOX-inducible) EPO-Tg gene construct. The transgene was configured with a tetracycline-responsive element (TRE) that drives the expression of the Epo gene. (B) Epo gene levels in the kidney (left), EPO protein levels in the serum (middle), and hematocrit levels (right) at 2 weeks after DOX feeding in Epo-Tg rtTA+ and rtTA- control mice. (C) Albumin/creatinine in the urine at the end of 3 weeks of aristolochic acid treatment followed by 3 weeks of follow-up. ( $\mathbf{D}$ and $\mathbf{F})$ Representative plots and (E and $\mathbf{G})$ data quantification of splenic IL-17+CD4+ $4^{+}$Th17 cells and FOXP3 ${ }^{+} \mathrm{CD} 4^{+}$Tregs. (H) Ratio between Treg and Th17 cells ( $n=3-7$ per group). ${ }^{*} P<0.05$, unpaired $t$ test. Data represent mean \pm SEM.

Endogenous and rEPO reduces Th17 cells and increases Tregs, while lessening disease severity in pristane-induced lupus. To test whether EPO inhibits/reverses Th17 cell-associated autoimmunity in another kidney disease model, we next studied pristane-induced nephritis in B6 mice $(28,29)$, a kidney disease model that mimics some aspects of lupus nephritis and is known to be driven by Th17 cell immunity (30). One week after initiating disease in B6 mice, we observed lower Epo mRNA expression in pristane- versus control-treated animals $(0.09 \pm 0.08$ vs. $0.92 \pm 0.10$ fold expression; $P<0.05)(29)$. Previous studies by others indicated that EPO/EPO-R signaling on monocyte/macrophages modulates pristane-induced nephropathy by promoting clearance of apoptotic cells, whose accumulation elicits an autoimmune response (12). An effect on $\mathrm{T}$ cells was not previously addressed. To test whether endogenous EPO binding to $\mathrm{T}$ cellexpressed EPO-R modulates Th17 cell development in this system, we administered pristane to EPO-R $\mathrm{R}^{\mathrm{f} / \mathrm{fl}}$ $\times \mathrm{CD} 4-\mathrm{Cre}^{+}$mice (which lack EPO-R on all T cells) and to EPO- $\mathrm{R}^{\mathrm{f} / \mathrm{fl}}$ controls, testing for the clinical and immunological evidence of lupus nephritis 2 months later. Whereas renal Epo gene expression (and serum levels) was similar between the EPO- $\mathrm{R}^{\mathrm{f} / \mathrm{f}} \mathrm{CD} 4-\mathrm{Cre}^{+}$mice and the EPO- $\mathrm{R}^{\mathrm{f} / \mathrm{fl}}$ controls (Figure 7, A and B), the EPO- $\mathrm{R}^{\mathrm{A} / \mathrm{f}} \mathrm{CD} 4-\mathrm{Cre}^{+}$mice showed more albuminuria, more severe histological lesions of lupus nephritis (Figure 7, C and D), and increased intrarenal expression of Rorc and Il17 genes (Figure 7, E and F).

In a converse strategy, we tested whether administration of rEPO dampens the clinical and immunological manifestation of pristane-induced nephropathy. We administered $\mathrm{rEPO}$ (or vehicle control) at 2 
A
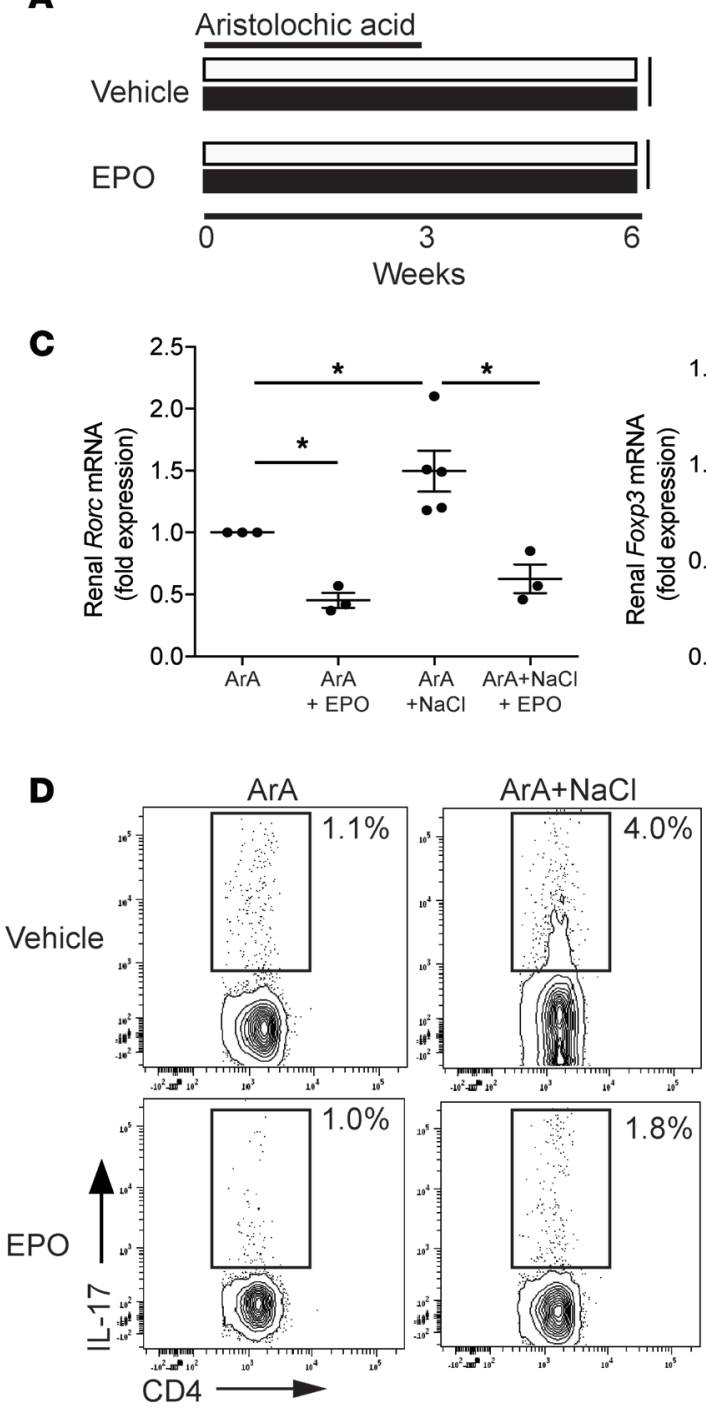

$\mathbf{E}$
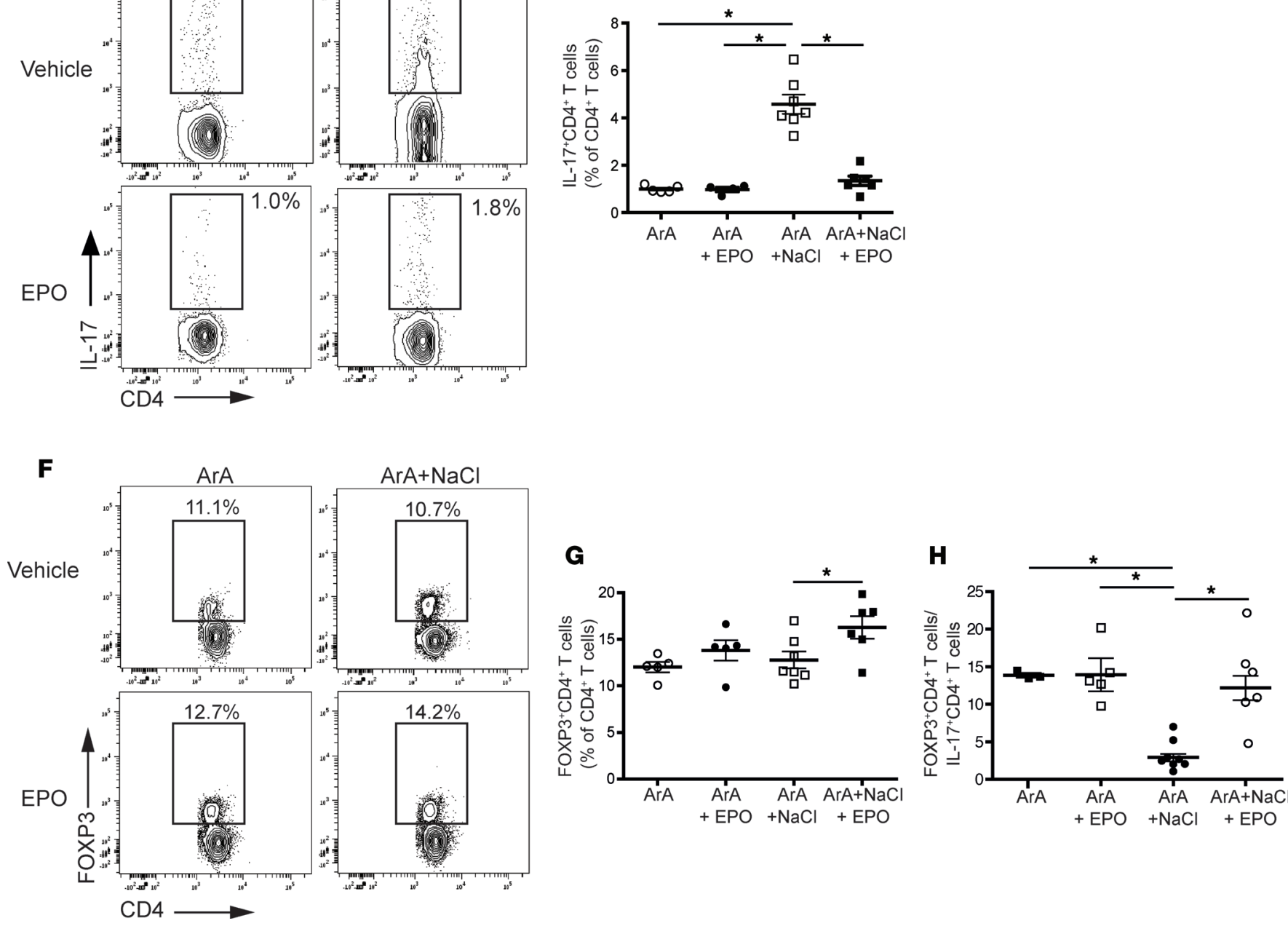

Figure 6. Recombinant EPO prevents NaCl-driven Th17 cell increase in aristolochic acid model. Male B6 mice were treated with aristolochic acid for 3 weeks and control or high-salt diet. Half of the animals received EPO and half received vehicle control (in A schematic of the experimental design; $n=4-7$ per group). (B) Urinary albumin/creatinine (ACR; ELISA) and (C) intrarenal Rorc (left) and Foxp3 (right) mRNA expression (qRT-PCR) at 6 weeks after the first ArA administration. ( $D$ and $\mathbf{F})$ Representative plots and data quantification (E and $\mathbf{G})$ of splenic IL-17+CD4+ Th17 cells and FOXP3 ${ }^{+}$CD4 ${ }^{+}$Tregs. (H) Ratio between Tregs and Th17 cells. ${ }^{*} P<0.05,2$-way ANOVA with Tukey test. Data represent mean \pm SEM. 
A

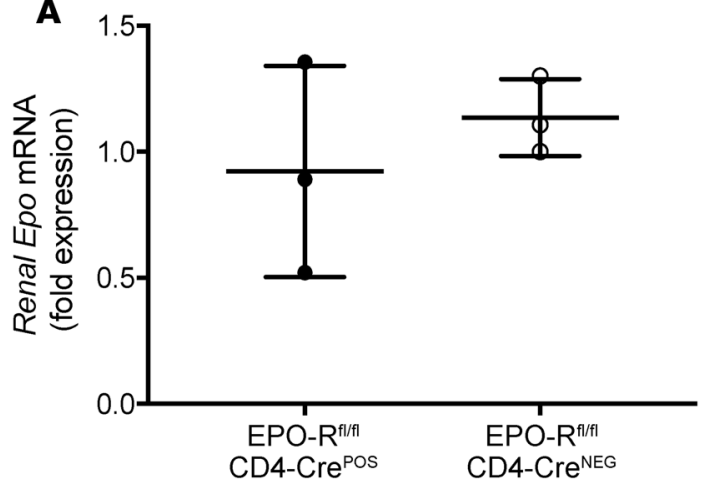

C

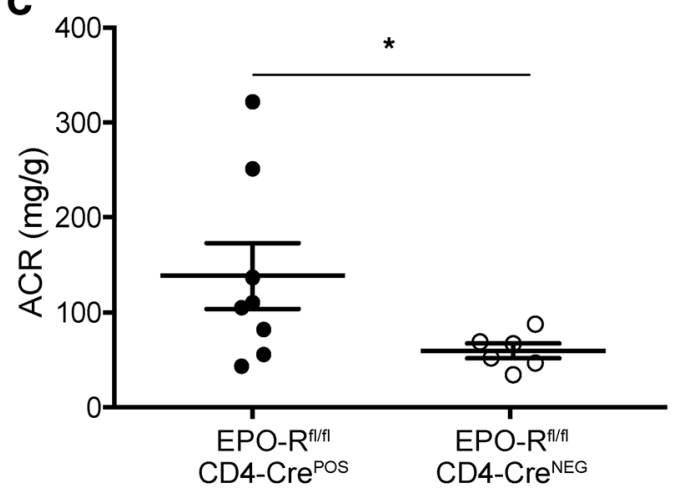

B

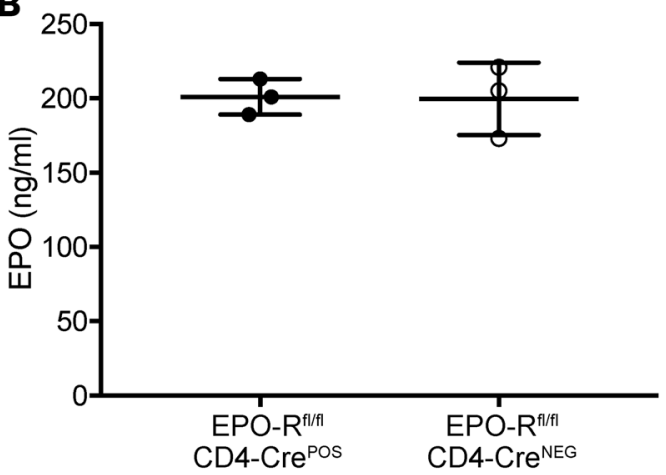

D

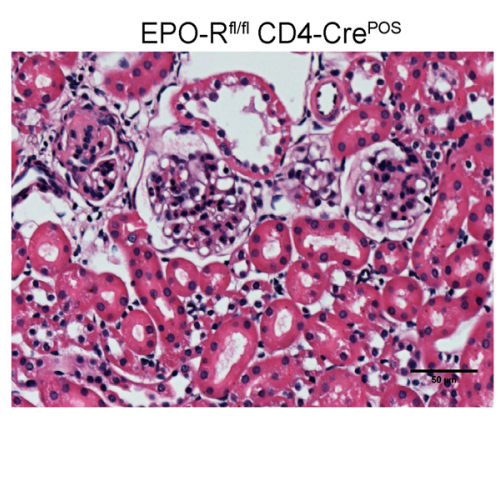

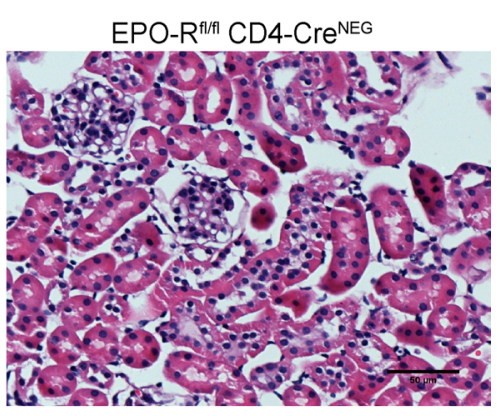

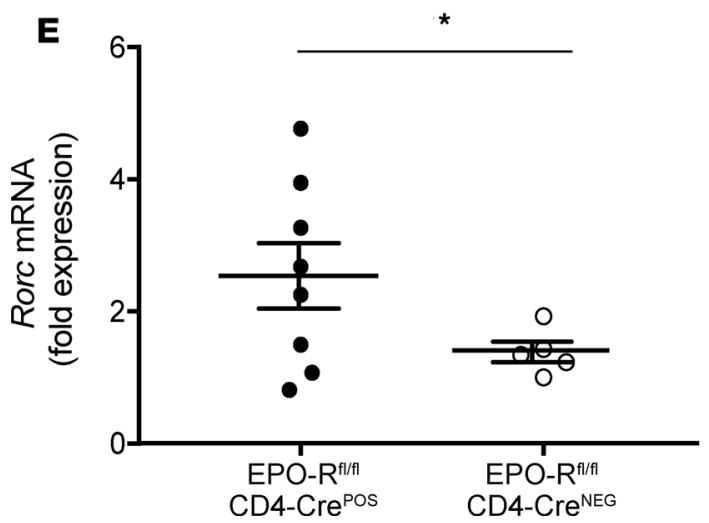

$\mathbf{F}$

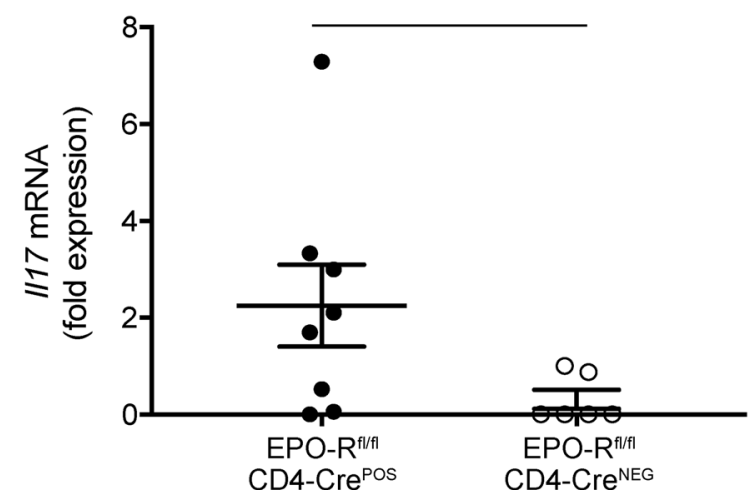

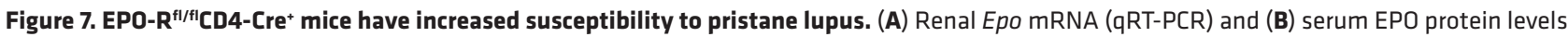

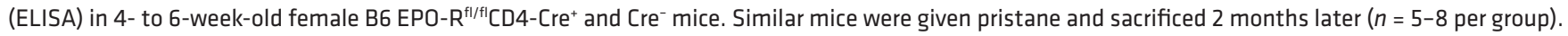
(C) Urinary albumin/creatinine (ACR; by ELISA) and (D) representative H\&E-stained sections (original magnification, $\times 20$ ). (E and F) Rorc and I/17 mRNA by qRT-PCR of kidney tissue from EPO- $\mathrm{R}^{\mathrm{fl} / \mathrm{f}} \mathrm{CD} 4-\mathrm{Cre}{ }^{+}$and $\mathrm{Cre}^{-}$mice. ${ }^{*} P<0.05$, unpaired $t$ test. Data represent mean $\pm \mathrm{SEM}$.

months after pristane injection and followed the animals for an additional 2 months (until 4 months after pristane injection). These experiments showed that, whereas the vehicle-treated mice developed albuminuria, circulating autoantibodies, and histological features of lupus nephritis, rEPO administration significantly reduced these signs of disease severity (Figure 8, A-G, and Supplemental Figure 9) and resulted in lower frequencies of Th17 cells with higher frequencies of FOXP3 ${ }^{+}$Tregs (Figure 8, H-K).

To test the hypothesis that $\mathrm{rEPO}$ improves disease severity and Th17 cell formation by directly signaling through $\mathrm{T}$ cell-expressed EPO-R, we treated EPO- $\mathrm{R}^{\mathrm{f} / \mathrm{l}} \mathrm{CD} 4-\mathrm{Cre}^{+}$and $\mathrm{Cre}^{-}$mice with $\mathrm{rEPO}$ or saline for 3 weeks after pristane injection and measured IL- $17^{+} \mathrm{CD} 4^{+} \mathrm{T}$ cells in the spleen thereafter. The data show that, while rEPO reduced Th17 cells in control mice, it had no effect on Th17 cells in mice where EPO-R was absent selectively on $\mathrm{T}$ cells (Figure $8, \mathrm{~L}$ and $\mathrm{M}$ ).

rEPO reduces disease severity in MRL-lpr lupus. To test EPO's ability to inhibit Th17 cell-induced kidney disease in a third model system, we studied MRL-lpr mice that spontaneously develop a lupus-like, Th17 
A

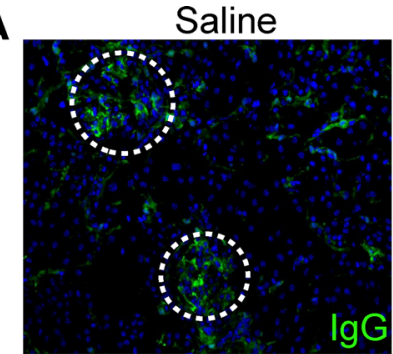

B

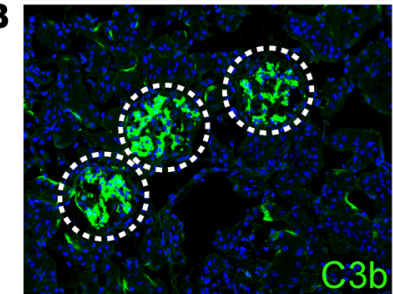

C

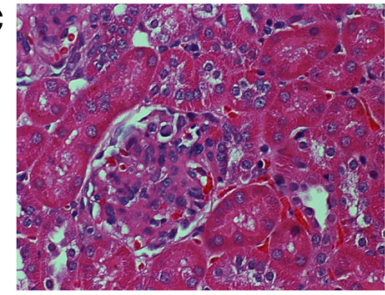

H

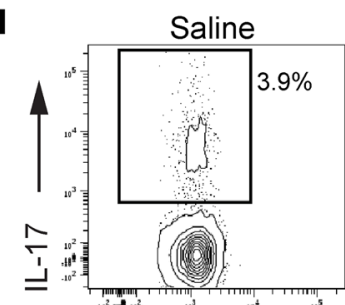

J

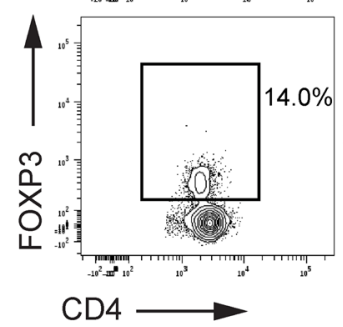

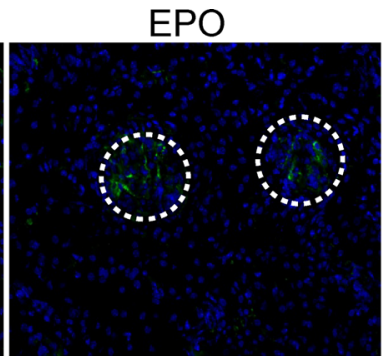
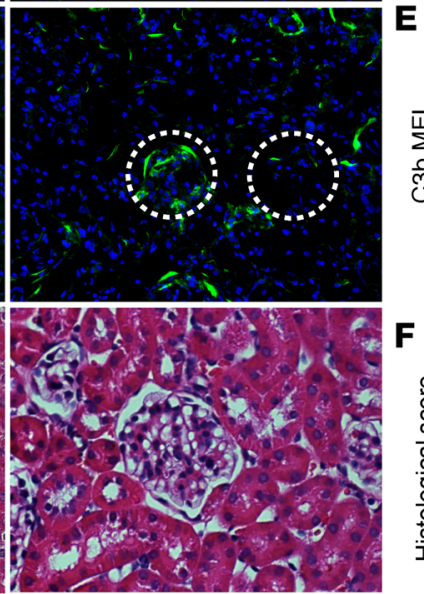

\section{$\mathbf{F}$}
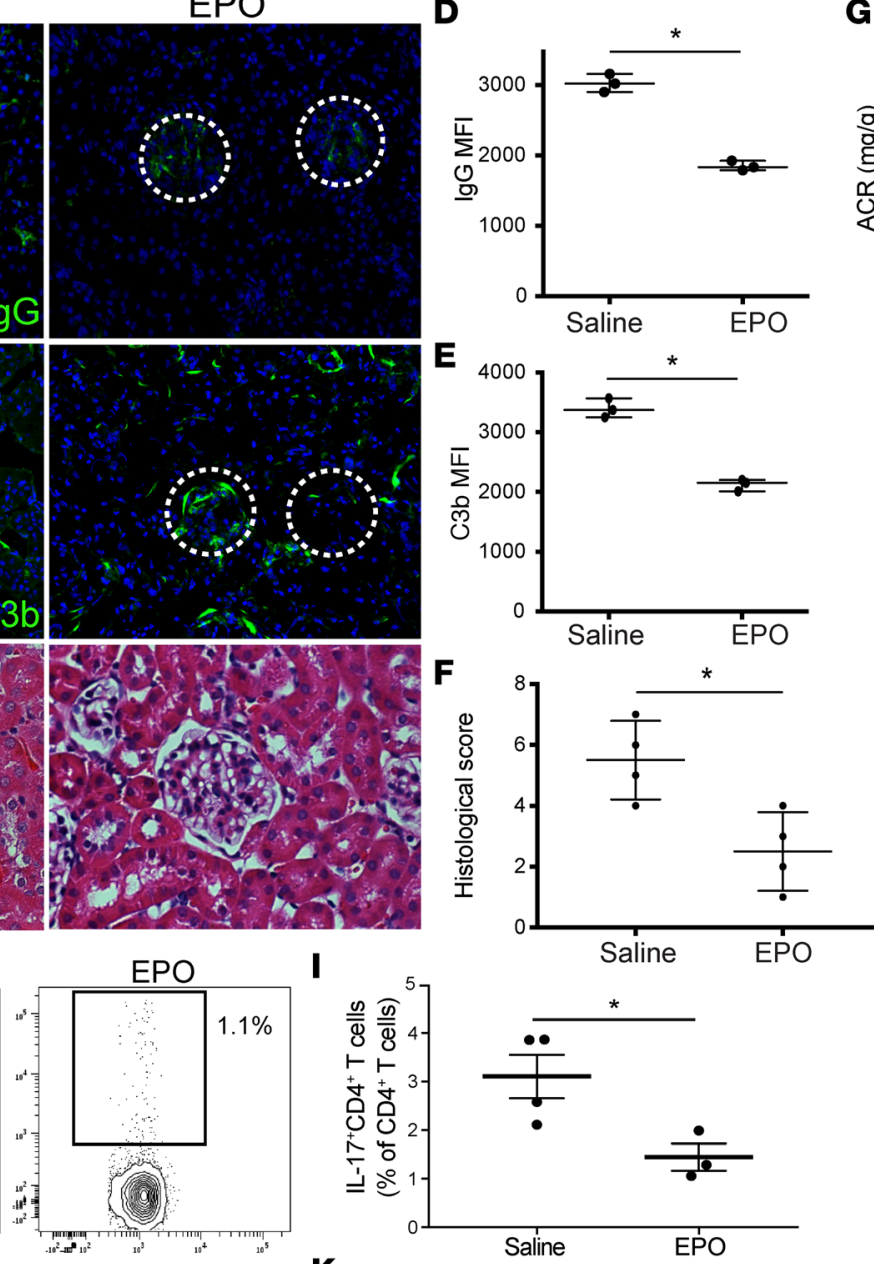

I
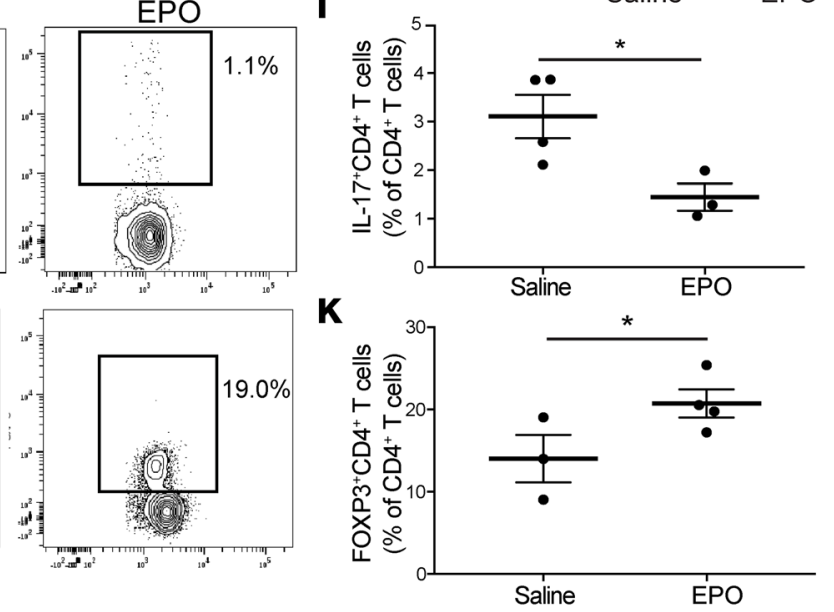

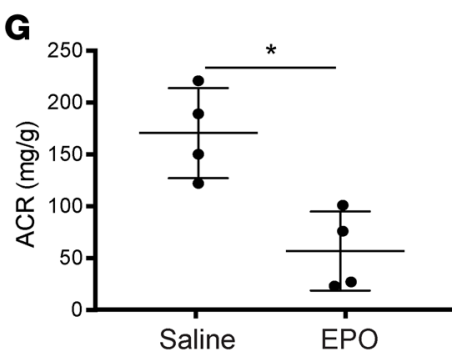

Figure 8. EPO ameliorates severity of pristane-induced murine lupus by inhibiting Th17 cells and increasing Tregs. B6 male mice ( $n=3-4$ per group) were treated with pristane at the age of 2 months and either EPO or vehicle control for 2 additional months and then sacrificed. Representative images of (A) IgC and (B) C3b glomerular deposition and (C) H\&E staining of the kidneys. Original magnification, $\times 20$ (A and $\mathbf{B}$ ); $\times 40$ (C). Differences in (D) IgC and (E) C3b glomerular fluorescent intensity between EPO- and vehicle-treated animals were quantified relative to DAPI using MetaMorph software. At least 30 glomeruli from 3 animals were included in the analysis. (F) Difference in total histological score between EPO- and vehicle-treated animals. (C) Urinary albumin/creatinine ratio (ACR). (H and J) Representative plots and (I and $\mathbf{K}$ ) data quantification of splenic $\mathrm{IL}-17^{+} \mathrm{CD} 4^{+}$ Th17 cells and CD4+FOXP3 ${ }^{+}$Tregs. (L) Representative plots and (M) data quantification of splenic $\mathrm{CD} 4^{+} \mathrm{IL}-17^{+}$ Th17 cells in EPO- $\mathrm{R}^{\mathrm{fl} / \mathrm{f}} \mathrm{CD} 4-\mathrm{Cre}^{+}$and Cre- male mice at 3 weeks after pristane injection ( $n=4-7$ per group). ${ }^{*} P<0.05$, unpaired $t$ test or 2-way ANOVA with Tukey test. Data represent mean \pm SEM.

\section{$\mathbf{L}$}
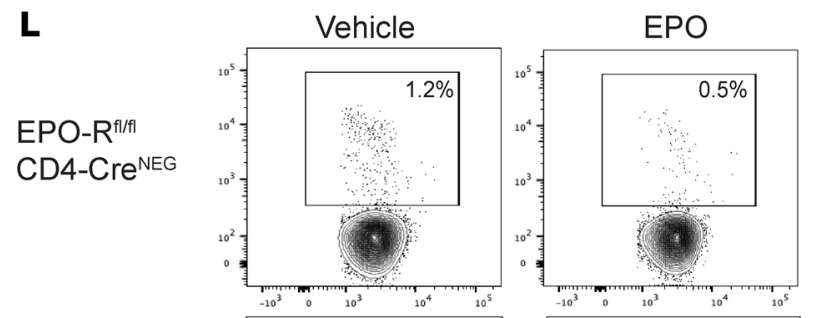

EPO-R ${ }^{\text {fl/fil }}$ CD4-Cre POS

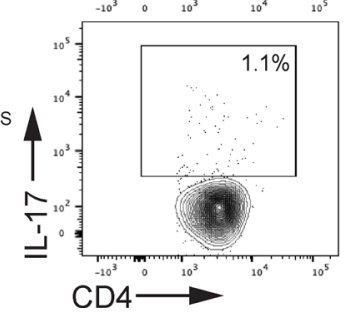

M

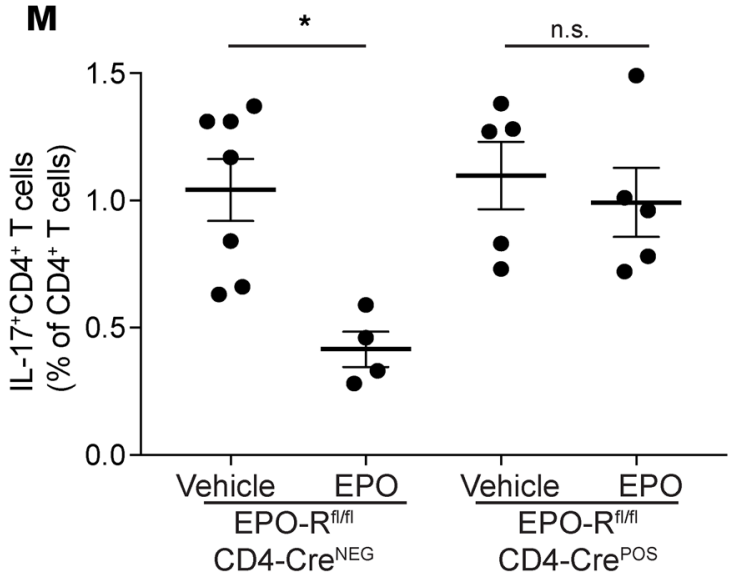


A

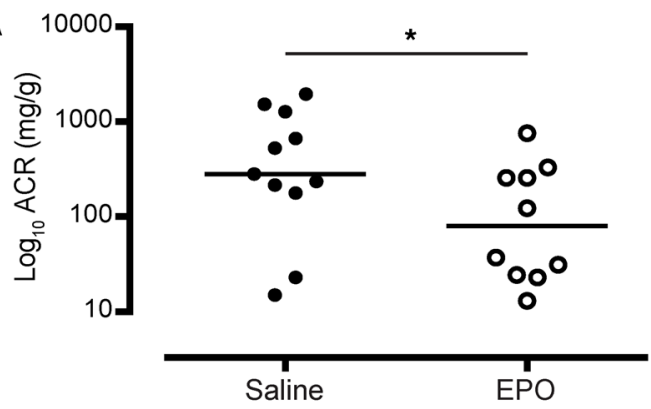

B

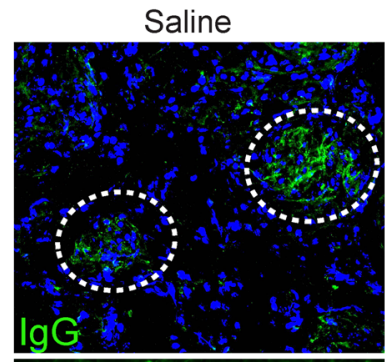

c

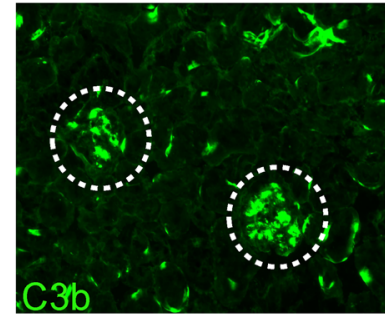

D

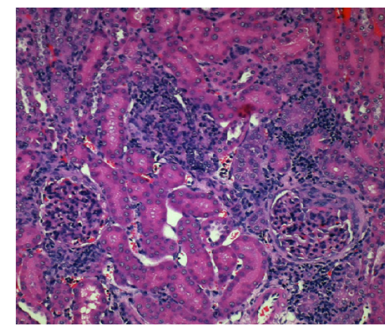

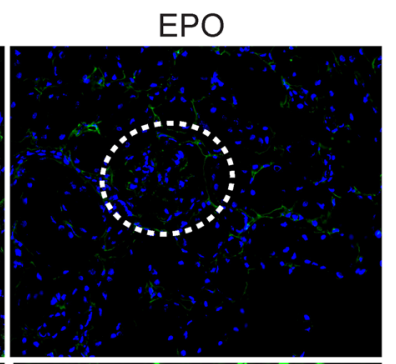
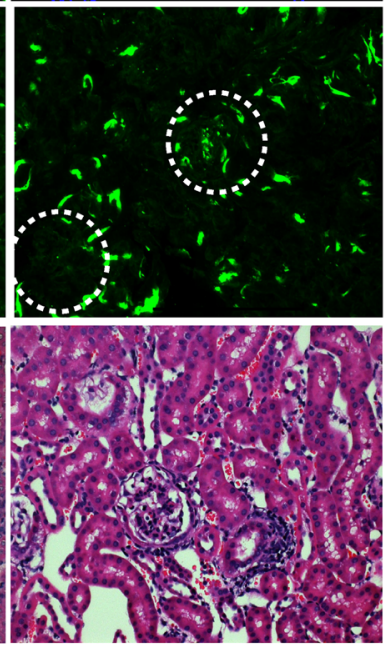

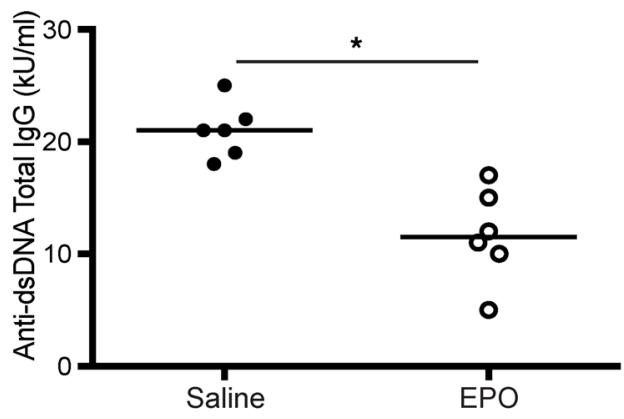

E
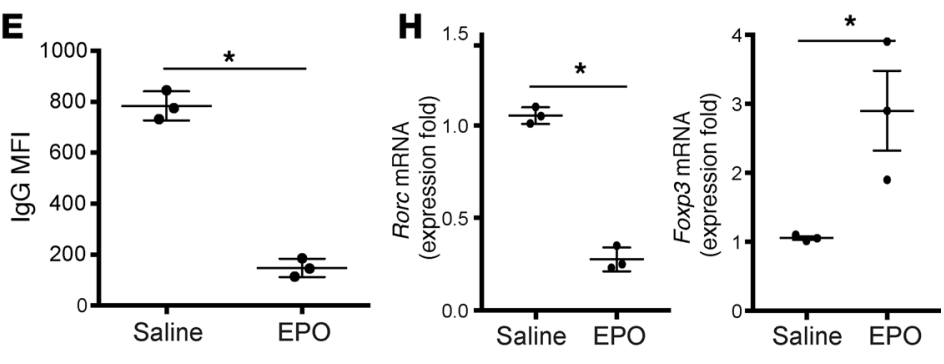

G
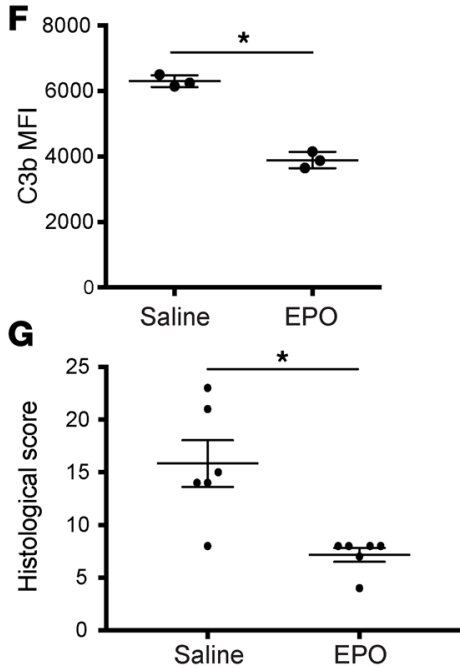

I
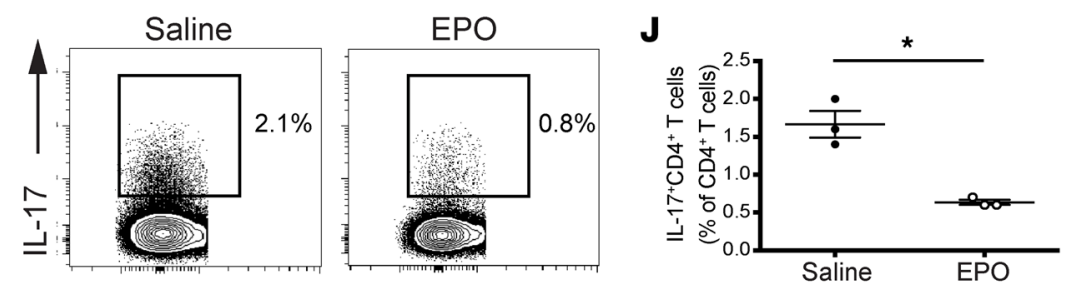

K
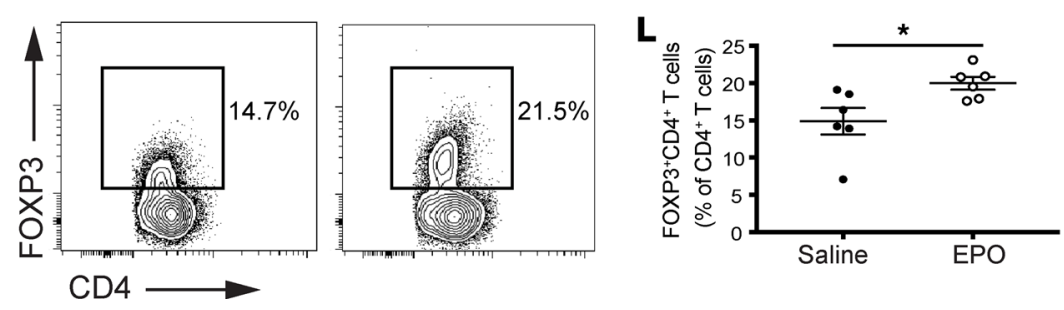

Figure 9. EPO ameliorates severity of lupus in MRL-Ipr mice by inhibiting Th17 cells and increasing Tregs. (A) Urinary albumin/creatinine ratio (ACR, left) and anti-dsDNA autoantibodies (right, ELISA) in 4-month-old MRL-Ipr mice treated with saline or rEPO from the age of 2 months ( $n=6-9$ per group) (median). (B) Representative IgG and (C) C3b in the renal glomeruli and (D) H\&E staining of intrarenal infiltrates (original magnification, $\times 20$ ). Differences in (E) IgG and (F) C3b glomerular fluorescent intensity between EPO- and vehicle-treated animals. (G) Difference in total histological score between EPO- and vehicle-treated animals. (H) Foxp3 and Rorc mRNA in kidney tissue (qRT-PCR). (I and K) Representative plots and (J and L) data quantification of splenic $\mathrm{IL}-17^{+} \mathrm{CD} 4{ }^{+}$Th17 cells and FOXP3+CD4+ Tregs. ${ }^{*} P<0.05$, unpaired $t$ test. Data represent mean \pm SEM. 
cell-dependent kidney disease as a consequence of a mutation in the Fas gene (31). We treated MRL-lpr mice with rEPO or vehicle control for 2 months beginning at 2 months of age, a time when the animals begin to spontaneously develop albuminuria and antibody deposition in the glomeruli (32). Whereas the control mice developed severe albuminuria, high titers of circulating anti-dsDNA autoantibodies (Figure 9A), positive glomerular staining for $\mathrm{IgG}$ and $\mathrm{C} 3 \mathrm{~b}$, and diffuse intrarenal mononuclear cell inflammatory infiltrates (Figure 9, B-G), rEPO treatment significantly reduced/limited of these disease parameters (Figure 9, A-G, and Supplemental Figure 10). When we performed qRT-PCR analyses of the kidneys from treated and control mice, we observed significantly less Rorc and more Foxp3 gene expression in the rEPO-treated animals (Figure 9H). Analysis of splenic T cells showed significantly fewer Th17 cells and more FOXP3 ${ }^{+}$Tregs in the EPO-treated animals (Figure 9, I-L). EPO treatment also reduced the frequencies of IFN- $\gamma$-producing CD4 ${ }^{+} \mathrm{T}$ cells (Supplemental Figure 11).

\section{Discussion}

While thymic education eliminates high-affinity self-reactive $\mathrm{T}$ cells, additional peripheral tolerance mechanisms are critical to prevent pathogenic immune responses directed against environmental antigens and self-antigens, particularly at mucosal sites (33). Similar to the gastrointestinal tract, where local Treg induction is crucial to control immune responses against gastrointestinal microbes and dietary antigens (34), the kidney is a mucosal tissue constantly exposed to environmental antigens and resident microbiome (35-37), but counterregulatory mechanisms that inhibit these potentially pathogenic stimuli have not been clearly delineated. Collectively, we believe the data provided herein newly indicate that kidney-derived EPO functions in this capacity to modulate proinflammatory immune responses in the kidney. Our in vivo findings document that intrarenal EPO modulates the severity of multiple Th17 cell-associated murine disease models, prevents Th17 cell differentiation in vivo, and drives transdifferentiation of preformed Th17 cells into $\mathrm{CD}^{+} \mathrm{FOXP}^{+} \mathrm{T}$ cells.

Our mechanistic analyses using in vitro and in vivo systems show that EPO directly binds to T cellexpressed EPO-R to inhibit the SGK1 signaling axis, a crucial pathway for Th17 cell induction (38). During Th17 cell differentiation, transcription of $S G K 1$ increases (19) as does SGK1 activation by p38 (21). This leads to phosphorylation and inhibition of FOXO1, a direct repressor of IL-23R expression (19). Our studies demonstrate that EPO/EPO-R signaling inhibits $S G K 1$ transcription and restrains activation of p38 and SGK1. Together, the data provide evidence that EPO directly counteracts Th17 cell induction at the molecular level.

Our data from rag1 $1^{-/-}$mice injected with Th17 cells further document that EPO promotes transdifferentiation of Th17 cells into FOXP3 ${ }^{+} \mathrm{CD}^{+} \mathrm{T}$ cells. Although we did not test the suppressive function of these IL-17- cells, our previous data indicate that EPO-induced FOXP3 expression confers suppressive capacity (15), thereby supporting the concept that EPO facilitates conversion of Th17 cells into functional FOXP3 ${ }^{+}$ Tregs. This result explains and adds mechanistic insight into findings published by others (20) showing that SGK1 can negatively regulate Treg development and reciprocally regulates development of the Th17 cell/ Treg balance. Mechanistically, SGK1 induces phosphorylation of FOXO1, resulting in the cytoplasmic retention of FOXO1, which inhibits the transactivation and maintenance of FOXP3. By inhibiting SGK1, EPO twists the Th17 cell/Treg balance toward increased Tregs.

Importantly, our in vivo data show that intrarenal EPO the regulates severity of renal disease in 3 Th17 cell-mediated murine models. Similar to Balkan nephropathy in humans, the clinically relevant ArA nephropathy model is characterized by reduced EPO expression, tubulointerstitial fibrosis, and T cell infiltrates (27). In this model, both transgenically increased endogenous EPO and administration of rEPO improved disease severity and reduced intrarenal expression of Rorc and Il17 genes and peripheral Th17 cells. The renal inflammation observed in this disease seems therefore driven or largely sustained by the reduced EPO levels, a consequence of the ArA-induced damage to EPO-producing cells $(16,17)$. Together with previous data by others (39) showing that EPO confers protection against acute tubular damage and attenuated interstitial fibrosis in a mouse model of ArA nephropathy, our findings provide a rationale for studies testing $\mathrm{rEPO}$ as a therapeutic strategy to treat renal and immune abnormalities in subjects with Balkan nephropathy. In this model, we also documented that $\mathrm{NaCl}$, while representing a major driver for RORC expression and Th17 cell differentiation, also promotes EPO production by the kidney $(40,41)$. While the 2 mechanisms are likely to be independent, our data suggest that $\mathrm{NaCl}$-driven EPO production may counteract further Th17 cell induction. 
We also demonstrate that EPO inhibits Th17 cell immunity and ameliorates disease severity in pristane-induced nephritis and in MRL-lpr mice, models of lupus nephritis characterized by elevations in Th17 cell immunity along with defects in Tregs (42). While previous data by others have shown that rEPO treatment prevents disease progression in both models, to our knowledge this has never been linked to a direct effect of EPO on T cells $(12,43)$. We believe our data newly demonstrate that lupus nephritis is associated with reduced Epo gene expression and rEPO treatment prevents Th17 cell induction and increases the Treg/Th17 cell ratio, a previously unrecognized effect targeting a crucial pathogenic mechanism (44).

Lack of EPO-R in T cells increases susceptibility to pristane-induced lupus in B6 mice, a strain that is relatively resistant to the development of disease (45), further supporting the importance of endogenous EPO in directly controlling Th17 cell formation through EPO-R signaling. Importantly, mice selectively lacking EPO-R in T cells are resistant to EPO inhibitory effects on Th17 cells, further indicating that EPO also directly inhibits Th17 cell induction in this in vivo model. Reduced EPO concentration or the presence of antibodies against EPO or EPO-R are common in human systemic lupus erythematosus (46) and correlate with disease severity $(47,48)$, along with percentages of Th17 cells (49), supporting a causal link among low EPO/EPO-R signaling, Th17 cell induction, and systemic lupus erythematosus activity in affected patients

Collectively, our data provide an explanation for previous studies reporting EPO-induced amelioration of experimental autoimmune encephalomyelitis, a mouse model of multiple sclerosis, in which Th17 cells play a pathogenic role (50). Similarly, EPO treatment has been shown to reduce the severity of optic neuritis or multiple sclerosis in humans (51-53), possibly as a consequence of its inhibitory effects on Th17 cells. While some of these results are possibly independent from the immunological effects of EPO, it is reasonable to speculate that others are, at least in part, linked to EPO-induced inhibition of autoreactive Th17 cells.

Our recently identified immune-modulating effects of EPO may be also responsible for the prolonged graft survival in renal transplant patients treated with high doses of rEPO (54) and are consistent with the high expression of EPO mRNA in renal allografts of immunologically tolerant cynomolgus monkeys (55) and with the reported association between EPO-R transcripts in peripheral blood mononuclear cells from kidney transplant recipients and acute rejection (56). Immune modulating effects of EPO may also be, at least in part, responsible for the its beneficial effects in patients on dialysis (57), whose chronic inflammatory state has been linked to increased cardiovascular risk (58).

The balance between Th17 cells and Tregs has emerged as a prominent factor in regulating cancer as well (59), and the fact that EPO shifts the ratio in favor of Tregs may contribute to the accelerated cancer progression described in patients receiving rEPO therapy (60).

In conclusion, our in vitro and in vivo data describe a previously unrecognized effect of EPO in counteracting Th17 cell induction and promoting the conversion of Th17 cells into Tregs. While these findings may have therapeutic implications for autoimmune diseases, organ transplantation, and cancers, they also raise the intriguing idea that EPO, traditionally considered to be primarily important for red blood cell differentiation, plays a role in preserving peripheral tolerance in the kidney.

\section{Methods}

Isolation of human peripheral blood cell and T cell subsets. Peripheral blood mononuclear cells were isolated from buffy coats obtained from deidentified healthy donors (New York Blood Bank) through Ficoll-Hypaque (MilliporeSigma) density gradient centrifugation. $\mathrm{CD}^{+} \mathrm{CD} 45 \mathrm{RA}^{+} \mathrm{CD} 45 \mathrm{RO}^{-}$naive $\mathrm{T}$ cells were enriched through magnetic isolation using AutoMACS (Miltenyi Biotech) (>95\% purity).

Th17 cell induction. Naive CD4 ${ }^{+} \mathrm{T}$ cells were cultured in X-VIVO20 medium (Lonza) supplemented with $50 \mathrm{U} / \mathrm{ml}$ penicillin/streptomycin (MilliporeSigma), $50 \mu \mathrm{M}$ 2- $\beta$-mercaptoethanol (Gibco), $1 \%$ human $\mathrm{AB}$ serum (Gemini), $2 \mathrm{mM}$ L-glutamine (MilliporeSigma), 1\% Sodium Pyruvate (Corning), and 1\% Non-Essential Amino Acids (MilliporeSigma). Additional human cytokines used were recombinant active TGF-ß1 (10 ng/ml, Miltenyi Biotec), IL-6 (30 ng/ml, Cell Application), IL-23 (100 ng/ml, ProSpec), IL-1 $\beta$ (10 ng/ml, Cell Application), and IL-21 (50 ng/ml, Cell Application). We also added anti-IFN- $\gamma$ ( $4 \mu \mathrm{g} / \mathrm{ml}, \mathrm{BD}$ Biosciences) and anti-IL-4 (4 $\mu \mathrm{g} / \mathrm{ml}$ BD Biosciences). Cells were activated with anti-CD3/ anti-CD28 mAb beads ( $1 \mu \mathrm{g} / \mathrm{ml}, \mathrm{BD}$ Biosciences) and cultured with or without $\mathrm{NaCl}(20-40 \mathrm{mM})$ or urea (40-80 mM) with or without EPO (1000 IU/ml) for 5 days and collected for intracellular cytokine staining and flow cytometry and stained for apoptosis markers (annexin V/7-AAD, BD Pharmingen). RNA was collected at 24 hours to measure gene expression profile by real-time PCR. 
In some experiments, we used anisomycin (10 $\mu \mathrm{g} / \mathrm{ml}$, MilliporeSigma) or GSK650394 (10 nM, Tocris) to activate $\mathrm{p} 38$ or inhibit SGK1, respectively.

For Th17 cell induction with murine cells, naive $\mathrm{CD} 4^{+} \mathrm{T}$ cells were obtained from the spleen and lymph nodes and cultured for 5 days with TGF- $\beta 1$ ( $5 \mathrm{ng} / \mathrm{ml}$, BioXcell) and IL-6 (30 ng/ml, Peprotech).

Flow cytometry. For surface staining, human cells were stained using standard approaches as published previously (15). Antibodies to human antigens were obtained from BD Pharmingen: APC-Cy7-anti-CD4 (clone RPA-T4), PE-anti-CD8 (clone RPA-T8), APC-Cy7-anti-CD8 (clone SK1), Fixable Viability Dye eFluor 450, and APC-anti-CD25 (clone M-A251). To measure intracellular cytokines, cells were stimulated for 5 hours at $37^{\circ} \mathrm{C}$ with PMA (100 ng/ml, MilliporeSigma) and ionomycin $(100 \mathrm{ng} / \mathrm{ml}$, MilliporeSig$\mathrm{ma}$ ) in the presence of GolgiStop (BD Biosciences). Fixation and permeabilization were performed using Cytofix/Cytoperm Plus (BD Biosciences), followed by staining with the appropriate antibodies, including FITC-anti-FOXP3 (clone HIL-7R-M21), PE-anti-IL-17A (clone N49-653), and PE-anti-IFN- $\gamma$ (clone B27). Antibodies to mouse antigens were obtained from BD Pharmingen: APC-Cy7-anti-CD4 (clone GK1.5), Pacific Blue-anti-CD8 (clone RPA-T8) and APC-anti-CD25 (clone PC61), PE-anti-FOXP3 (clone MF23), PE-anti-IL-17A (clone TC11-18H10), and PE-anti-IFN- $\gamma$ (clone XMG1.2).

For phospho-flow signaling analyses, human $\mathrm{T}$ cells were distributed into round-bottom 96-well plates $\left(2 \times 10^{5}\right.$ /each well) in RPMI Free Serum Media (Gibco) and allowed to rest at $37^{\circ} \mathrm{C}$ for 4 hours. Cells were kept resting or activated with anti-CD3/anti-CD28 mAb beads $\left(25 \mu 1 / 10^{6}\right)$ and $\mathrm{NaCl}(20 \mathrm{mM})$ or urea $(40$ $\mathrm{mM})$ with or without $\mathrm{EPO}(1000 \mathrm{IU} / \mathrm{ml})$ for 0,30 , and 60 minutes at $37^{\circ} \mathrm{C}$. The reaction was stopped by fixation with $4 \%$ paraformaldehyde (Affymetrix) for 10 minutes at $37^{\circ} \mathrm{C}$. The cells were washed with PBS and permeabilized by resuspension in $90 \%$ cold methanol (Fisher Scientific) for 15 minutes in the rocker. Samples were washed 3 times with PBS and 1\% Fetal Bovine Serum (Thermo Scientific) and stained overnight at $4^{\circ} \mathrm{C}$ for Pacific Blue anti-CD4 (clone RPA-T4, BD Pharmingen), pSGK1 (Ser422, Thermo Fisher), and PE-pp38 (Thr180, Tyr182, clone 4NIT4KK, eBioscience).

Data were acquired (10,000-100,000 events) on a 3-laser Canto II flow cytometer (BD Biosciences) and analyzed using CytoBank (https://www.cytobank.org) or FlowJo (https://www.flowjo.com) software.

Real-time quantitative reverse transcription PCR. RNA was prepared from $0.5 \mathrm{~cm}$ of the mouse kidneys or from cultured naive $\mathrm{CD}^{+} \mathrm{T}$ cells at day 3 of culture in Th17 cell-polarizing conditions using Trizol (Invitrogen). cDNA was synthesized using reverse transcription reagent (Applied Biosystems). Real-time PCR assays using the TaqMan universal PCR Master Mix and primer sets for human and mouse EPO (Mm01202755 $\mathrm{m} 1)$, RORC (Hs01076112_m1; Mm01261022_m1), FOXP3 (Mm00475162_m1), SGK1 (Hs00178612_m1), IL23R (Hs00332759_m1), FOXO1 (Hs00231106_m1), IL17A (Hs00174383_m1; Mm00439618_m1), Rn18S (Hs03928992_g1; Mm03928990_g1), and GAPDH(Hs02786624_g1; Mm99999915_g1) genes were purchased from Thermo Fisher. PCR was performed on an Applied Biosystems 7500 Fast system. All experiments were performed at least in triplicate, and gene expression was normalized to housekeeping gene 18 s or GAPDH.

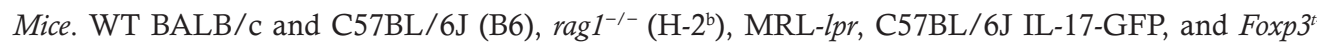
m3(DTR/GFP) $\left(\right.$ Foxp $\left.3^{D T R}\right)$ mice were purchased from The Jackson Laboratory. C57BL/6J EPO-Tg mice were obtained by standard techniques through the mouse genetics core facility at Mount Sinai and were crossed to mice expressing the rtTA transgene under the ROSA promoter (The Jackson Laboratory). C57BL/6J EPO- $\mathrm{R}^{\mathrm{f} / \mathrm{fl}}$ mice were obtained from ES cells from the Knockout Mouse Project (Davis, California, USA). We produced and screened funders at Mount Sinai core facility, and then we crossed them with CD4-Cre ${ }^{+/-}$ mice (The Jackson Laboratory).

Procedures. Adoptive transfer of flow-sorted IL-17-GFP ${ }^{+} \mathrm{CD} 4{ }^{+} \mathrm{T}$ cells or naive $\mathrm{CD} 44^{\text {lo }} \mathrm{CD} 62 \mathrm{~L}^{\text {hi }} \mathrm{CD} 4{ }^{+}$ $\mathrm{T}$ cells into $\mathrm{rag} 1^{-/-}$mice was done through a retro-orbital injection.

ArA (MilliporeSigma) was administered i.p. at the dose of $3 \mathrm{mg} / \mathrm{kg} 3$ times per week for 3 weeks. Pristane (MilliporeSigma) was administered as a single i.p. 0.5-ml dose. DT (MilliporeSigma) was administered at the dose of $10 \mu \mathrm{g} / \mathrm{kg}$ i.p. 3 times per week. Human rEPO $\alpha$ (Procrit) was obtained from Janssen-Cilag.

Renal histology. Animals were deeply anesthetized and transcardially perfused with periodate-lysine-paraformaldehyde fixate at $4 \%$ in PBS, and the kidneys were dissected out. Kidney samples were either frozen in Optimal Cutting Temperature (O.C.T., Tissue-Tek) compound or fixed in 10\% formalin and then embedded in paraffin.

Light microscopy. Formalin-fixed paraffin-embedded kidney sections $(3 \mu \mathrm{m})$ were stained with $\mathrm{H} \& \mathrm{E}$ and Masson's trichrome to highlight interstitial fibrosis in the ArA model. Histological scoring was performed in a blinded manner by a renal pathologist according to a previously established method (61). 
Immunofluorescence analysis in renal tissue. Samples were frozen in O.C.T. compound. First, cryosections (5- $\mu \mathrm{m}$ thick) were incubated with PBS for 5 minutes and then with blocking solution containing $5 \%$ bovine serum albumin for 30 minutes at room temperature. For renal tissue markers, the following antibodies were used: goat FITC-conjugated anti-mouse C3b (1:100; Cappel) for 1 hour at room temperature and anti-mouse IgG antibody conjugated with Alexa Fluor 488 (1:500; Jackson Immunoresearch) for 1 hour at room temperature. Nuclei were counterstained with DAPI (Invitrogen). At least 20 glomeruli per section for each animal were randomly acquired using a fluorescence nonconfocal laser scanning microscope (Zeiss Axio Imager Z2 M). Surface antibodies expression was estimated by constructing a contour mask on the bright-field image. Software ImageJ (NIH) was used to quantify C3b and IgG staining intensity.

EPO and urine albumin/creatinine measurement. To quantify proteinuria, we measured urine creatinine and urine albumin concentration to calculate UACR. Urine creatinine was quantified using the Creatinine (urinary) Colorimetric Assay Kit from Cayman Chemical. Urine albumin was determined using a commercial assay from Bethyl Laboratory Inc. Urine albumin excretion was expressed as the ratio of urine albumin to creatinine.

The concentration of EPO serum samples was measured using the Mouse Erythropoitin ELISA Kit from Abcam according to the manufacturer's recommendations.

Statistics. We used paired or unpaired 2-tailed $t$ tests for 2-group comparisons and 1-way or 2-way ANOVA (with Tukey test for post hoc pairwise differences) for multiple independent group comparisons. A 2-tailed $P$ value of less than 0.05 was regarded as statistically significant. All statistical analyses were performed using GraphPad Prism (version 7 for Windows, GraphPad Software Inc.).

Study approval. Study protocols were approved by the Institutional Animal Care and Use Committee at the Icahn School of Medicine at Mount Sinai.

\section{Author contributions}

$\mathrm{CD}, \mathrm{AA}$, and CC performed most of the experiments, did the data analyses, and reviewed the manuscript. VDD performed histological scoring. GLM and EF helped in data interpretation and critically revised the manuscript. JKH helped in the experiments with EPO-Tg and -knockdown mice. HX helped in the experiments with IL-17-GFP mice. CG performed experiments with p38 and SGK1 inhibitors. SH helped with experiments with EPO-R conditional-KO mice and took care of the mice. JCM helped with data interpretation and critically revised the manuscript. UM supervised statistical analyses. PSH generated the EPO-Tg mice, critically helped with interpretation of the results, and reviewed/edited the manuscript. PC conceived and oversaw the project, interpreted the results, and wrote the manuscript.

\section{Acknowledgments}

This work was supported by the National Institutes of Health National Institute of Allergy and Infectious Diseases grant R01 0255A141 awarded to PC and grant P01 AI123086 awarded to JCM and PSH. The content is solely the responsibility of the authors and does not necessarily represent the official views of the National Institutes of Health.

Address correspondence to: Paolo Cravedi, Icahn School of Medicine at Mount Sinai, 1 Levy Place, 10029 New York, New York, USA. Phone: 212.241.3349; Email: paolo.cravedi@mssm.edu.

1. Harrison OJ, Powrie FM. Regulatory T cells and immune tolerance in the intestine. Cold Spring Harb Perspect Biol. 2013;5(7):null.

2. Pabst O, Mowat AM. Oral tolerance to food protein. Mucosal Immunol. 2012;5(3):232-239.

3. Hall JA, Grainger JR, Spencer SP, Belkaid Y. The role of retinoic acid in tolerance and immunity. Immunity. 2011;35(1):13-22.

4. Caprioli F, Pallone F, Monteleone G. Th17 immune response in IBD: A new pathogenic mechanism. J Crohns Colitis. 2008;2(4):291-295.

5. Weaver CT, Elson CO, Fouser LA, Kolls JK. The Th17 pathway and inflammatory diseases of the intestines, lungs, and skin. Annu Rev Pathol. 2013;8:477-512.

6. Shilling RA, Wilkes DS. Role of Th17 cells and IL-17 in lung transplant rejection. Semin Immunopathol. 2011;33(2):129-134

7. Paust HJ, et al. The IL-23/Th17 axis contributes to renal injury in experimental glomerulonephritis. J Am Soc Nephrol. 2009;20(5):969-979

8. Amarilyo G, Lourenço EV, Shi FD, La Cava A. IL-17 promotes murine lupus. J Immunol. 2014;193(2):540-543.

9. Pindjakova J, et al. Interleukin-1 accounts for intrarenal Th17 cell activation during ureteral obstruction. Kidney Int 2012;81(4):379-390.

10. Broxmeyer HE. Erythropoietin: multiple targets, actions, and modifying influences for biological and clinical consideration. J Exp Med. 2013;210(2):205-208. 
11. Bunn HF. Erythropoietin. Cold Spring Harb Perspect Med. 2013;3(3):a011619.

12. Luo B, et al. Erythropoeitin Signaling in Macrophages Promotes Dying Cell Clearance and Immune Tolerance. Immunity. 2016;44(2):287-302

13. Mausberg AK, et al. Erythropoietin ameliorates rat experimental autoimmune neuritis by inducing transforming growth factor- $\beta$ in macrophages. PLoS One. 2011;6(10):e26280.

14. Cravedi P, et al. Immunosuppressive effects of erythropoietin on human alloreactive T cells. J Am Soc Nephrol. 2014;25(9):2003-2015

15. Purroy C, et al. Erythropoietin Receptor-Mediated Molecular Crosstalk Promotes T Cell Immunoregulation and Transplant Survival. J Am Soc Nephrol. 2017;28(8):2377-2392.

16. Pavlović-Kentera V, Clemons GK, Trbojević S, Dimković N, Djukanović L. Erythropoietin and anemia in the progression of Balkan endemic nephropathy and other renal diseases. Nephron. 1990;54(2):139-143.

17. Pavlović-Kentera V, Djukanović L, Clemons GK, Trbojević S, Dimković N, Slavković A. Anemia in Balkan endemic nephropathy. Kidney Int Suppl. 1991;34:S46-S48.

18. Kleinewietfeld M, et al. Sodium chloride drives autoimmune disease by the induction of pathogenic TH17 cells. Nature. 2013;496(7446):518-522.

19. Wu C, et al. Induction of pathogenic TH17 cells by inducible salt-sensing kinase SGK1. Nature. 2013;496(7446):513-517.

20. Wu C, et al. SGK1 Governs the Reciprocal Development of Th17 and Regulatory T Cells. Cell Rep. 2018;22(3):653-665.

21. Zhou X, Ferraris JD, Dmitrieva NI, Liu Y, Burg MB. MKP-1 inhibits high NaCl-induced activation of p38 but does not inhibit the activation of TonEBP/OREBP: opposite roles of p38alpha and p38delta. Proc Natl Acad Sci USA. 2008;105(14):5620-5625

22. Downs-Canner S, et al. Suppressive IL-17A(+)Foxp3(+) and ex-Th17 IL-17A(neg)Foxp3(+) Treg cells are a source of tumour-associated Treg cells. Nat Commun. 2017;8:14649.

23. Gagliani N, et al. Th17 cells transdifferentiate into regulatory T cells during resolution of inflammation. Nature. 2015;523(7559):221-225.

24. Jadot I, Declèves AE, Nortier J, Caron N. An Integrated View of Aristolochic Acid Nephropathy: Update of the Literature. Int J Mol Sci. 2017;18(2):297.

25. Luciano RL, Perazella MA. Aristolochic acid nephropathy: epidemiology, clinical presentation, and treatment. Drug Saf. 2015;38(1):55-64

26. Staneva $\mathrm{R}$, et al. Whole genome methylation array analysis reveals new aspects in Balkan endemic nephropathy etiology. BMC Nephrol. 2013;14:225.

27. Zhou Y, Bian X, Fang L, He W, Dai C, Yang J. Aristolochic acid causes albuminuria by promoting mitochondrial DNA damage and dysfunction in podocyte. PLoS One. 2013;8(12):e83408.

28. Reeves WH, Lee PY, Weinstein JS, Satoh M, Lu L. Induction of autoimmunity by pristane and other naturally occurring hydrocarbons. Trends Immunol. 2009;30(9):455-464.

29. Leiss $\mathrm{H}$, et al. Pristane-induced lupus as a model of human lupus arthritis: evolvement of autoantibodies, internal organ and joint inflammation. Lupus. 2013;22(8):778-792.

30. Mardani F, Mahmoudi M, Esmaeili SA, Khorasani S, Tabasi N, Rastin M. In vivo study: Th1-Th17 reduction in pristane-induced systemic lupus erythematosus mice after treatment with tolerogenic Lactobacillus probiotics. J Cell Physiol. 2018;234(1):642-649.

31. Santiago-Raber ML, Laporte C, Reininger L, Izui S. Genetic basis of murine lupus. Autoimmun Rev. 2004;3(1):33-39

32. Mountz JD, Gause WC, Jonsson R. Murine models for systemic lupus erythematosus and Sjögren's syndrome. Curr Opin Rheumatol. 1991;3(5):738-756.

33. Xing Y, Hogquist KA. T-cell tolerance: central and peripheral. Cold Spring Harb Perspect Biol. 2012;4(6):a006957.

34. Sun CM, et al. Small intestine lamina propria dendritic cells promote de novo generation of Foxp3 T reg cells via retinoic acid. JExp Med. 2007;204(8):1775-1785.

35. Rani A, et al. Urinary microbiome of kidney transplant patients reveals dysbiosis with potential for antibiotic resistance. Transl Res. 2017;181:59-70.

36. Ling Z, Liu F, Shao L, Cheng Y, Li L. Dysbiosis of the Urinary Microbiota Associated With Urine Levels of Proinflammatory Chemokine Interleukin-8 in Female Type 2 Diabetic Patients. Front Immunol. 2017;8:1032.

37. Liu F, et al. Dysbiosis of urinary microbiota is positively correlated with type 2 diabetes mellitus. Oncotarget. 2017;8(3):3798-3810.

38. Wu H, et al. High salt promotes autoimmunity by TET2-induced DNA demethylation and driving the differentiation of Tfh cells. Sci Rep. 2016;6:28065.

39. Hamano Y, et al. Low-dose darbepoetin alpha attenuates progression of a mouse model of aristolochic acid nephropathy through early tubular protection. Nephron Exp Nephrol. 2010;114(2):e69-e81.

40. Yanai H, Katsuyayama H. A Possible Mechanism for Renoprotective Effect of Sodium-Glucose Cotransporter 2 Inhibitor: Elevation of Erythropoietin Production. J Clin Med Res. 2017;9(2):178-179.

41. Donnelly S. Why is erythropoietin made in the kidney? The kidney functions as a 'critmeter' to regulate the hematocrit. Adv Exp Med Biol. 2003;543:73-87.

42. Yang H, Wang H, Shivalila CS, Cheng AW, Shi L, Jaenisch R. One-step generation of mice carrying reporter and conditional alleles by CRISPR/Cas-mediated genome engineering. Cell. 2013;154(6):1370-1379.

43. Huang B, et al. Non-erythropoietic erythropoietin-derived peptide protects mice from systemic lupus erythematosus. J Cell Mol Med. 2018;22(7):3330-3339.

44. Yang J, et al. Th17 and natural Treg cell population dynamics in systemic lupus erythematosus. Arthritis Rheum. 2009;60(5):1472-1483.

45. Richard ML, Gilkeson G. Mouse models of lupus: what they tell us and what they don't. Lupus Sci Med. 2018;5(1):e000199.

46. Voulgarelis M, Kokori SI, Ioannidis JP, Tzioufas AG, Kyriaki D, Moutsopoulos HM. Anaemia in systemic lupus erythematosus: aetiological profile and the role of erythropoietin. Ann Rheum Dis. 2000;59(3):217-222.

47. Luo XY, et al. Anti-erythropoietin receptor antibodies in systemic lupus erythematosus patients with anemia. Lupus. 2013;22(2):121-127 
48. Hara A, et al. Effect of Autoantibodies to Erythropoietin Receptor in Systemic Lupus Erythematosus with Biopsy-proven Lupus Nephritis. J Rheumatol. 2016;43(7):1328-1334.

49. Krebs CF, Schmidt T, Riedel JH, Panzer U. T helper type 17 cells in immune-mediated glomerular disease. Nat Rev Nephrol. 2017;13(10):647-659.

50. Yuan R, Maeda Y, Li W, Lu W, Cook S, Dowling P. Erythropoietin: a potent inducer of peripheral immuno/inflammatory modulation in autoimmune EAE. PLoS One. 2008;3(4):e1924.

51. Sühs KW, et al. Disease Activity and Conversion into Multiple Sclerosis after Optic Neuritis Is Treated with Erythropoietin. Int JMol Sci. 2016;17(10):null.

52. Suhs KW, et al. A randomized, double-blind, phase 2 study of erythropoietin in optic neuritis. Annals of neurology 2012;72(2):199-210.

53. Borhani-Haghighi A, et al. Erythropoietin for acute multiple sclerosis in patients with optic neuritis as a first demyelination event. Neurosciences (Riyadh). 2012;17(2):151-155.

54. Choukroun G, et al. Correction of postkidney transplant anemia reduces progression of allograft nephropathy. $J$ Am Soc Nephrol. 2012;23(2):360-368

55. Smith RN, et al. RNA expression profiling of renal allografts in a nonhuman primate identifies variation in NK and endothelial gene expression. Am J Transplant. 2018;18(6):1340-1350.

56. Roedder S, et al. The kSORT assay to detect renal transplant patients at high risk for acute rejection: results of the multicenter AART study. PLoS Med. 2014;11(11):e1001759.

57. Locatelli F, Becker H. Update on anemia management in nephrology, including current guidelines on the use of erythropoiesis-stimulating agents and implications of the introduction of "biosimilars". Oncologist. 2009;14 Suppl 1:16-21.

58. Tripepi G, Mallamaci F, Zoccali C. Inflammation markers, adhesion molecules, and all-cause and cardiovascular mortality in patients with ESRD: searching for the best risk marker by multivariate modeling. J Am Soc Nephrol. 2005;16 Suppl 1:S83-S88.

59. Knochelmann HM, et al. When worlds collide: Th17 and Treg cells in cancer and autoimmunity. Cell Mol Immunol. 2018;15(5):458-469.

60. Debeljak N, Solár P, Sytkowski AJ. Erythropoietin and cancer: the unintended consequences of anemia correction. Front Immunol. 2014;5:563.

61. Bajema IM, et al. Revision of the International Society of Nephrology/Renal Pathology Society classification for lupus nephritis: clarification of definitions, and modified National Institutes of Health activity and chronicity indices. Kidney Int. 2018;93(4):789-796. 